Pacific Journal of Mathematics

LINEAR FUNCTIONALS ON ORLICZ SPACES: GENERAL 


\title{
LINEAR FUNCTIONALS ON ORLICZ SPACES: GENERAL THEORY
}

\author{
M. M. RAO
}

Let $\Phi$ be a generalized Young's function and $L^{\Phi}$ the corresponding Orlicz space, on a general measure space. The problem considered here is the characterization of the dual space $\left(L^{\Phi}\right)^{*}$, in terms of integral representations, without any further restrictions. A complete solution of the problem is presented in this paper. If $\Phi$ is continuous and the measure space is sigma finite (or localizable), then a characterization of the second dual $\left(L^{\Phi}\right)^{* *}$ is also given. A detailed account of the quotient spaces of $L^{\Phi}$ relative to certain subspaces is presented; and the analysis appears useful in the study of such spaces as the Riesz and Köthe-Toeplitz spaces.

The purpose of this paper is two-fold. First it contains a complete study centering around the singular linear functionals, analyzing certain factor (or quotient) spaces, of the Orlicz spaces. Second, the so-called 'generalized Young's functions' and the associated Orlicz spaces, and their adjoint spaces, are also considered. (Precise definitions will be given later.) The work here is a continuation of [19] and the notation and terminology of that paper will be maintained. However, the theory presented here subsumes [19], and the exposition is essentially self-contained.

If $\Phi$ and $\Psi$ are complementary Young's functions (cf. Definition 1 below), let $L^{\Phi}$ and $L^{\Psi}$ be the corresponding Orlicz spaces on a (not necessarily finite or even localizable) measure space $(\Omega, \Sigma, \mu)$ which has only the (nonrestrictive) finite subset property. This latter means that every set of positive $\mu$-measure has a subset of positive finite $\mu$-measure. Then the representation problem for continuous linear functionals on $L^{\Phi}$ is to express them as integrals relative to appropriate additive set functions on $\Sigma$. In [1] and [19] certain general integral representations of such elements were obtained when the Young's function $\Phi$ and the measure $\mu$ satisfy some restrictions. If $\mathscr{l}^{\Phi}$ is the closed subspace of $L^{\Phi}$ spanned by the $u$-step functions then $x^{*} \in\left(L^{\oplus}\right)^{*}$, adjoint of $L^{\oplus}$, is termed singular if $x^{*}\left(\mathscr{C l}^{\Phi}\right)=0$, i.e., $x^{*} \in\left(\mathscr{C}^{\varphi}\right)^{\perp}$, and it is absolutely continuous if there exists a $\chi_{E} \in \mathscr{C}^{\Phi}$, and $x^{*}\left(\chi_{E}\right) \neq 0$, where $\chi_{E}$ is the indicator of $E \in \Sigma$. It is known that $\left(\mathscr{C l}^{\Phi}\right)^{\perp} \neq\{0\}$ if $\Phi$ is continuous and grows exponentially fast. In Theorem 2 (and hence 3) of [19] it was announced that every $x^{*} \in\left(L^{\Phi}\right)^{*}$ is of the form $x^{*}(f)=\int_{\Omega} f d G$ for a certain additive set function $G$. However, the result was proved only for such $x^{*}$ that $x^{*}\left(\chi_{E}\right) \neq 0$ for 
at least one $\chi_{E} \in \mathscr{M}^{\Phi}$, and the case that $x^{*} \in\left(\mathscr{C}^{\Phi}\right)^{\perp}$ was inadvertently left out. Thus Theorems 2 and 3 of [19] must be understood as results on representing absolutely continuous linear functionals on $L^{\oplus}$ in the sense defined above where $\Phi$ is a Young's function. The result is restated precisely in Theorem 1 below for completeness. Moreover, it is shown later (Proposition 2) that $\left(L^{\Phi}\right)^{*}$ admits a direct sum decomposition consisting of the singular and the absolutely continuous elements. Also the generalized (or the extended from the original version of) Young's functions are considered in detail below. This distinction is irrelevant for the elementary theory of Orlicz spaces (such as completeness, etc.), or if the underlying measure $\mu$ is finite, but will be relevant for the study of the adjoint spaces and the understanding of their structures when $\mu$ is nonfinite. This will be clear from the work of $\S 3$ and $\S 4$ below.

The main contribution of this paper is the complete characterization of $\left(L^{\Phi}\right)^{*}$, the adjoint space of $L^{\Phi}$, when both $\Phi$ and $\mu$ are general. This is achieved by considering, somewhat more generally, the properties of certain factor spaces of $L^{\Phi}$ which in particular illuminate the structure of singular linear functionals. Also the $\left(L^{\Phi}\right)^{*}$-space when $\Phi$ is a 'generalized Young's function' is considered and characterized. Moreover, the general methods presented here are applicable in analyzing the more general Banach function spaces such as the Köthe-Toeplitz spaces [12] and their extensions [15]. A brief summary of the results is as follows.

After preliminaries in the next section, representation theorems, extending the work of [1] and [19], are proved in $\S 3$. These results are further extended, when $\Phi$ is a generalized Young's function, in $\S 4$. Some miscellaneous results are given in $\S 5$ where the current status of the work, and the representation of the elements of $\left(L^{\phi}\right)^{* *}$, and related problems are discussed.

2. Preliminaries. It is convenient to start with some definitions. The following is essentially the concept introduced in [24]. (cf. [27] and [13].)

Definition 1. A symmetric convex function $\Phi$ defined on the line with $\Phi(0)=0$ is said to be a Young's function if it is either discontinuous, i.e., $\Phi(x)$ is continuous for $0 \leqq|x| \leqq x_{1}, \Phi(x)=\infty$ for $|x|>x_{1}$, or, if continuous satisfies $\Phi(t)>0$ for $t>0$. A function $\Psi$ is said to be (Young's) complementary function to $\Phi$ and is defined by

$$
\Psi(x)=\sup \{|x| y-\Phi(y): y \geqq 0\},-\infty<x<\infty .
$$

It is seen that $\Psi(\cdot)$ is a symmetric convex function on the line 
with $\Psi(0)=0$, but if $\Psi$ is continuous it may fail to be a Young's function in the above sense. However it can be described by

Definition 2. A symmetric continuous convex function $\Phi$, with $\Phi(0)=0$, defined on the line is said to be a generalized Young's function, if $\Phi(x)=0$, for $0 \leqq x \leqq a$, for some finite positive number a.

Note that a discontinuous generalized Young's function and the discontinuous (ordinary) Young's function are the same. (However, the trivial $\Phi$, taking only 0 and $+\infty$, will be excluded.) Thus the complementary Young's function can be a generalized Young's function (as simple examples show). Clearly (2.1) implies the Young's inequality

$$
x y \leqq \Phi(x)+\Psi(y) .
$$

There is equality if and only if either $x=\Psi^{\prime}(y)$ or $y=\Phi^{\prime}(x)$. [ $\left[\Phi^{\prime}, \Psi^{\prime}\right.$ stand for the right derivatives of $\Phi, \Psi$ which exist everywhere (cf. [13], p. 5), and which are nondecreasing and right continuous.] In what follows the above terminology will be used without further mention. Thus $(\Phi, \Psi)$ denote complementary (generalized) Young's functions. Some needed preliminary results will be proved in this section.

The set $L^{\Phi}$ is a subspace of all measurable real functions on $(\Omega, \Sigma, \mu)$ such that $f \in L^{\Phi}$ if and only if $\|f\|_{\Phi}<\infty$ or $N_{\Phi}(f)<\infty$ where

$$
\|f\|_{\Phi}=\sup \left\{\int_{\Omega}|f g| d \mu: \int_{\Omega} \Psi(g) d \mu \leqq 1\right\}
$$

and

$$
N_{\Phi}(f)=\inf \left\{k>0: \int_{\Omega} \Phi\left(\frac{f}{k}\right) d \mu \leqq 1\right\} .
$$

Then $\|\cdot\|_{\phi}$ and $N_{\phi}(\cdot)$ are norm functionals and they define the same topology for $L^{\Phi}$ in the sense that

$$
N_{\phi}(f) \leqq\|f\|_{\oplus} \leqq 2 N_{\phi}(f), \quad f \in L^{\phi} .
$$

With these norms $L^{\Phi}$ becomes a Banach (or $B$-) space. The proofs of these results may be found in ([13], [15], [26], or [27]). The set $\widetilde{L}^{\Phi}$ is the (not necessarily linear) class of functions $f \in L^{\Phi}$ for which $\int_{\Omega} \Phi(f) d \mu$ exists. [Strictly speaking, $L^{\Phi}$ is the space of equivalence classes of measurable functions and so $f \in L^{\Phi}$ means that $f$ is any member of the class to which it belongs.] All the results of this paper are valid for complex functions also.

In general the inequalities in (2.5) cannot be improved, as seen 
from the case that $\Phi(x)=|x|^{p}, p \geqq 1$. This is further illuminated by the following result.

Lemma 1. Let $\Phi$ and $\Psi$ be continuous Young's functions that are complementary. Then for $f \in L^{\Phi},\|f\|_{\Phi}=N_{\Phi}(f)$ if and only if $f=0$, a.e. $[\mu]$.

Proof. The 'if' being obvious, suppose conversely that $\|f\|_{\phi}=$ $N_{\phi}(f)$. If $f=0$, a.e., is false then $N_{\phi}(f)>0$ and it may be assumed, by a normalization if necessary, that $N_{\odot}(f)=1\left(=\|f\|_{\Phi}\right)$. Since $\Phi$ and $\Psi$ are continuous, a result of $([13]$, p. 92) is applicable and there exists a bounded sequence of numbers $\left\{k_{n}\right\}$ such that

$$
1=\lim _{n \rightarrow \infty} \frac{1}{k_{n}}\left(1+\int_{\Omega} \Phi\left(k_{n} f\right) d \mu\right) \text {. }
$$

So for any $\varepsilon>0$, there is an $n_{\varepsilon}$ such that $n \geqq n_{\varepsilon}$ implies

$$
(1+\varepsilon) k_{n} \geqq 1+\int_{\Omega} \Phi\left(k_{n} f\right) d \mu>1 .
$$

Thus $\lim _{n \rightarrow \infty} k_{n} \geqq(1+\varepsilon)^{-1}$. Since $\varepsilon>0$ is arbitrary $\lim _{n \rightarrow \infty} k_{n} \geqq 1$, so there exists a subsequence, which is again denoted by $\left\{k_{n}\right\}$ itself for convenience, such that in (2.7) $k_{n} \geqq 1$. If $k_{n}=1$, then (2.7) implies $\int_{\Omega} \Phi(f) d \mu \leqq \varepsilon$. But from the arbitrariness of $\varepsilon$, this implies $f=0$, a.e. (since $\Phi(x)>0$ for $x>0$ ), which is impossible. Thus $k_{n}>1$ for all large enough $n$. Consequently (2.7) gives, $\int_{0} \Phi(\alpha f) d \mu<\infty$ for some $\alpha>1$. From this result and ([27], p. 175, below eq. (10.20)) one can conclude that $\int_{\Omega} \Phi(f) d \mu=1$. Thus, the convexity of $\Phi$, and (2.7) imply

$$
\infty>(1+\varepsilon) k_{n} \geqq 1+k_{n} \int_{\Omega} \Phi(f) d \mu=1+k_{n}>2 .
$$

Since $\varepsilon$ is arbitrary one has from (2.8) the inequality

$$
\infty>\frac{\lim }{n \rightarrow \infty} k_{n} \geqq 1+\frac{\lim }{n \rightarrow \infty} k_{n} \geqq 2
$$

which is impossible. This shows that $f=0$, a.e. must be true, completing the proof.

REMarks. 1. This lemma says that, unless $\Phi$ or $\Psi$ is discontinuous, the inequalities in (2.5) are strict if $f \neq 0$, a.e.

2. If $\Phi$ and $\Phi^{\prime}$ are continuous then there exists a $k$ for which (2.6) holds (without the limit there) by ([13], pp. 92-93). It was 
proved in ([20], Th. 4) that under this hypothesis one always has $\int_{\Omega} \Phi\left(f / N_{\phi}(f)\right) d \mu=1$.

3. The structure of continuous Young's functions may be illuminated by the following remark: If $\Phi$ is a continuous Young's function then it is always possible to find a strictly convex Young's function $\Phi_{1}$ such tha $\Phi_{1}^{\prime}$ is strictly increasing (continuous) and $\Phi(x) \leqq \Phi_{1}(x) \leqq 2 \Phi(x)$, for all $x$ (so $L^{\Phi}$ and $L^{\Phi_{1}}$ are topologically equivalent). This follows from the constructions of Young's functions given in ([13], p. 21). In fact, let $\Phi_{1}^{\prime}(u)=\Phi^{\prime}(u)(1+\alpha(u))$, where $\alpha(\cdot)$ is a strictly increasing continuous (probability) distribution on the positive line, e.g. $\alpha(u)=$ $1-e^{-u}$, and let $\Phi_{1}(x)=\int_{0}^{|x|} \Phi_{1}^{\prime}(u) d \mu$. Clearly $\Phi_{1}$ satisfies the requirements stated above. Moreover, since $\Phi^{\prime}$ can be taken continuous by a redefinition (e.g., joining the discontinuities with straight line segments; cf. [27], p. 25), it follows that $\Phi_{1}^{\prime}$ is also continuous.

Let $\mathscr{C}^{\Phi}$ be the closed subspace of $L^{\Phi}$ spanned by all step functions. [A step function takes finitely many finite values on measurable sets, possibly of infinite measure.] Let $M^{\Phi}=\left\{f: \int_{\Omega} \Phi(k f) d \mu<\infty\right.$, all $\left.k>0\right\}$. Then $M^{\infty}$ is also a $B$-space (the same space with the same notation as introduced in [19]). The relation between these space is given by the following:

Lemma 2. $M^{\Phi} \cong \mathbb{C}^{\Phi} \cong L^{\Phi}$. There is equality between the first two spaces if $\Phi$ is a continuous Young's function. The last two spaces are equal if $\Phi$ is a discontinuous Young's function and $\mu(\Omega)<\infty$ or $\Phi$ is of the form $\Phi(x)=0$ for $0 \leqq x \leqq x_{0},=\Phi_{1}(x)$ for $x_{0} \leqq x \leqq x_{1}$ and $=\infty$ for $x>x_{1}($ and $\mu(\Omega) \leqq \infty)$ where $\Phi_{1}$ is a continuous convex function such that $\Phi_{1}(x)>0$ for $x>x_{0}$ and $x_{0}>0$.

Proof. The inclusions are clear. If the Young's function $\Phi$ is continuous then every step function in $L^{\Phi}$ is also a $\mu$-simple function (i.e., a step function that vanishes outside a set of finite $\mu$-measure), since every element of $\mathscr{L}^{\Phi}$ must vanish outside a set of $\sigma$-finite measure. So by ([19], Lemma 2) it follows that $M^{\Phi}=\mathscr{C}^{\Phi}$. (Note that if $\Phi$ does not satisfy a growth condition, [13], or grows exponentially fast then $\mathscr{L}^{\Phi} \varsubsetneqq L^{\Phi}$ holds. If $\Phi$ is discontinuous, then it is clear that $M^{\oplus}=\{0\}$ so that $M^{\oplus} \varsubsetneqq \mathscr{C}^{\Phi}$. This proves half of the lemma.

About the second equality, if $\Phi$ is discontinuous, it follows from the definition, of the norm (2.4), that every element of $L^{\Phi}$ is essentially bounded so that $L^{\Phi} \subset L^{\infty}$ always holds. If the discontinuous $\Phi$ is such that $\Phi(x)=0$, for $0 \leqq x \leqq x_{0}$ where $x_{0}>0$, then $L^{\Phi}=L^{\infty}$ since $f \in L^{\infty}$ implies, for some $k>0,0 \leqq(|f| / k) \leqq x_{0}$, a.e. [ $\left.\mu\right]$, so that $f \in L^{\Phi}$, by 
(2.4). If $x_{0}=0$ in the above and $\Phi$ is discontinuous (but is a positive convex function for $x \leqq x_{1}$ as in the lemma), and if $\mu(\Omega)<\infty$ then every $f$ in $L^{\infty}$ satisfies, $0 \leqq\left(|f| \mid k_{0}\right)<x_{1}$ for some $k_{0}>0$. Hence

$$
\int_{\Omega} \Phi\left(\frac{|f|}{k_{0}}\right) d \mu \leqq \Phi\left(x_{1}\right) \mu(\Omega)<\infty
$$

so that $f \in L^{\phi}$. Thus in both these cases $L^{\phi}=L^{\infty}$. Moreover every $f \in L^{\infty}$ satisfies (cf. (2.4) again) $\|f\|_{\infty} \leqq x_{1} N_{\phi}(f)$ where $\|\cdot\|_{\infty}$ is the essential supremum norm of $L^{\infty}$. Since $L^{\Phi}$ and $L^{\infty}$ are both $B$-spaces and contain the same elements it follows by an application of the closed graph theorem, on using the inequalities between the norms just noted, that the norms $\|\cdot\|_{\infty}$ and $N_{\phi}(\cdot)$ are equivalent (cf., [22], Ex. 2, p. 184) and hence also that $\|\cdot\|_{\infty}$ and $\|\cdot\|_{\infty}$ are equivalent, by (2.5). But step functions are dense in $L^{\infty}$ by ([6], p. 296). Since $L^{\infty}$ and $L^{\infty}$ are topologically equivalent under conditions of the lemma, proved above, it follows that $L^{\Phi}=\mathscr{C l}^{\Phi}$ in these cases. This completes the proof.

REMARK. The above proof shows that, if $\mu(\Omega)=\infty$, and $\Phi$ is discontinuous, $L^{\Phi} \varsubsetneqq L^{\infty}$ can happen only if $\Phi(x)>0$ for $0<x \leqq x_{1}$, and $=\infty$ for $x>x_{1}$. Note that the nonfiniteness of the measure space does interfere in the treatment here. For this $\Phi, \mathscr{C}^{\Phi}$ is just the closure of simple functions in $L^{\Phi}$ since then each step function is a simple function. Also if $\Phi$ is any continuous generalized Young's function, then $M^{\Phi} \varsubsetneqq \mathscr{C}^{\Phi}$ can happen. In this case $\Phi$ is not a Young's function and $L^{\infty} \varsubsetneqq \mathscr{C l}^{\Phi}$ holds, and in fact $\mathscr{C}^{\Phi}$ is then the $L^{\Phi}$-closure of $L^{\infty}$. It should also be remarked that in this case, $\mathscr{K}^{\Phi} \subset \widetilde{L}^{\Phi}$ holds only if $\mu(\Omega)<\infty$ in addition to the continuity of $\Phi$, since then $M^{\Phi}=\mathscr{C}^{\Phi}$. [Simple function = step function with support of finite measure.1

Motivated by the above lemma the set $L^{\Phi}-\mathscr{C l}^{\Phi}$ will now be examined. However, this set is not even linear (since the difference of two functions in it can belong to $\mathscr{C}^{\Phi}$ ) and moreover the structure of functions that are not in $\mathscr{C}^{\Phi}$ is desired. So it will be appropriate to consider $\mathscr{N}^{\Phi}=L^{\Phi} / \mathscr{L}^{\Phi}$, the factor or quotient space of $L^{\Phi}$ relative to $\mathscr{C}^{\Phi}$. Thus $f_{1}, f_{2}$ in $L^{\Phi}$ belong to the same equivalence class (or coset) in $\mathscr{N}^{\Phi}$ if and only if $f_{2}=f_{1}+g$, a.e., for some $g$ in $\mathscr{C}^{\Phi}$. The norm $d(\cdot)$ in $\mathscr{N}^{\Phi}$ is given by: if $\hat{f} \in \mathscr{N}^{\oplus}$ (i.e., $\hat{f}=f+\mathscr{C}^{\Phi}$, $\left.f \in L^{\varphi}\right)$ then

$$
d(\hat{f})=\inf \left\{\|f+g\|_{\Phi}: g \in \mathscr{C l}^{\Phi}\right\} .
$$

[Hereafter when no mention is made, $\Phi$ may be either an ordinary or a generalized Young's function.]

The mapping $\lambda: L^{\Phi} \rightarrow \mathscr{N}^{\Phi}$ is the canonical mapping of $L^{\Phi}$ onto 
$\mathscr{N}^{\Phi}$, and is continuous in the metric defined by (2.10). The following facts on factor spaces will be needed in the sequel.

LEMMA 3. (i) $\mathscr{N}^{\oplus}$ is a B-space with (2.10) as norm and $\lambda$ maps the open unit ball of $L^{\circ}$ onto the open unit ball of $\mathscr{N}^{\oplus}$. (ii) If $\left(\mathscr{C l}^{\Phi}\right)^{\perp}$ is the annihilator of $\mathscr{K}^{\Phi}$ (i.e., the set of elements of $\left(L^{\oplus}\right)^{*}$ that vanish on $\left.\mathscr{C l}^{\oplus}\right)$, then there is an isometric isomorphism of $\left(\mathscr{L}^{\oplus}\right)^{\perp}$ onto all of $\left(\mathscr{N}^{\oplus}\right)^{*}$ given by the mapping, $j: x^{*} \rightarrow z^{*}$ where $x^{*} \in\left(\mathscr{C l}^{\Phi}\right)^{\perp}$ and $z^{*} \in\left(\mathscr{N}^{\oplus}\right)^{*}$, the $z^{*}$ being defined by $z^{*}\left(f+\mathscr{M}^{\Phi}\right)=$ $x^{*}(f)$. [Thus, $x^{*} \in\left(\mathscr{C l}^{\oplus}\right)^{\perp}$ if and only if $\left.j\left(x^{*}\right)=z^{*} \in\left(\mathscr{N}^{*}\right)^{*}.\right]$

The statements (i) and (ii) are immediate consequences of the facts about factor spaces in general $B$-spaces ([6], p. 72; [22], p. 105). These properties clarify the structure of $\left(\mathscr{L}^{\Phi}\right)^{\perp}$. Because of (ii) of the lemma, $\left(\mathscr{C}^{\Phi}\right)^{\perp}$ and $\left(\mathscr{N}^{\Phi}\right)^{*}$ can and will be identified below, if there is no confusion. It will follow from results (Propositions 2, 3) in the next sections (after the introduction of some new concepts), that $\left(L^{\oplus}\right)^{*}$ admits a direct sum decomposition into complementary subspaces one of which is $\left(\mathscr{C l}^{\oplus}\right)^{\perp}$.

The first part of the above lemma for the particular space $\mathscr{N}^{\oplus}$ can be specialized as follows, and this is useful later.

Lemma 4. Let $L^{\Phi}$ be given the norm (2.3). Then $\lambda\left(\tilde{L}^{\Phi}\right)$ is mapped into the closed unit ball of $\mathscr{N}^{\oplus}$, and $\lambda\left(\widetilde{L}^{\Phi}\right)$ contains the open unit ball of $\mathscr{N}^{\Phi}$, where $\lambda: L^{\oplus} \rightarrow \mathscr{N}^{\oplus}$ is the canonical map and $\tilde{L}^{\Phi}$ was defined after (2.5). The inclusions above can be proper.

The proof of this lemma may be found in ([13], p. 82) for a finite nonatomic measure and for a continuous Young's function $\Phi$, where $L^{\Phi}$ has norm (2.3) as here. The same proof also holds verbatim for general $\mu$ and continuous $\Phi$. If $\Phi$ is discontinuous and $\mu$ is nonfinite, then the only case when $\mathscr{N}^{\Phi} \neq\{0\}$ is when $\Phi(x)>0$ for $0<x \leqq x_{1}$ and $=\infty$ for $x>x_{1}$ (where $\Phi(x)$ is continuous on $0<x<x_{1}$ ) as shown in the proof of Lemma 2 . In this case the proof that $\lambda\left(\widetilde{L}^{\Phi}\right)$ is mapped into the closed unit ball of $\mathscr{N}^{\bullet}$, given in ([13], p. 82), again holds without change as before. On the other hand, if $d(\hat{f})<1$, then, by definition, there is an $f \in \hat{f}$ such that $\|f\|_{\infty}<1$ and by (2.5) $N_{\Phi}(f)<1$. So $f \in \widetilde{L}^{\Phi}$ and $\hat{f} \in \lambda\left(\widetilde{L}^{\Phi}\right)$. Hence $\lambda\left(\widetilde{L}^{\Phi}\right)$ contains the open unit ball of $\mathscr{N}^{\oplus}$, as desired.

The lattice structure of the spaces $L^{\Phi}, \widetilde{L}^{\Phi}$ and $\mathscr{N}^{\oplus}$ is needed and it will be discussed now. It is clear that $L^{\oplus}$ is a Banach lattice, where the ordering $f \leqq g$ stands for $f \leqq g$, a.e. With this ordering $\widetilde{L}^{\Phi}$ is a lattice, since $f, g$ in $\widetilde{L}^{\Phi}$ implies clearly $\inf (f, g) \in \widetilde{L}^{\Phi}$, and if 
$h=\sup (f, g)$, then

$$
\int_{\Omega} \Phi(h) d \mu=\int_{\Omega_{1}} \Phi(f) d \mu+\int_{\Omega_{2}} \Phi(g) d \mu<\infty,
$$

where $\Omega_{1}=\{\omega: f(\omega) \geqq g(\omega)\}$ and $\Omega_{2}=\Omega-\Omega_{1}$, so that $h \in \tilde{L}^{\Phi}$. The quotient space $\mathscr{N}^{\Phi}\left(=L^{\Phi} / \mathscr{C}^{\Phi}\right)$ inherits a 'natural' ordering from that of $L^{\Phi}$. With respect to that ordering $\mathscr{N}^{\Phi}$ is also a vector lattice. The ordering and the proof that $\mathscr{N}^{\oplus}$ is a vector lattice follow.

If $\hat{f}, \hat{g}$ are the elements (cosets of $f, g$ in $L^{\Phi}$ ) in $\mathscr{N}^{\oplus}$ define $\hat{f} \leqq \hat{g}$ if there exist an $f_{0} \in \hat{f}$ and $g_{0} \in \hat{g}$ such that $f_{0} \leqq g_{0}$, a.e. It is clearly reflexive, and to see transitivity, let $\hat{f}, \hat{g}, \hat{h}$ be in $\mathscr{N}^{\oplus}$ such that $\hat{f} \leqq \hat{g}$ and $\hat{g} \leqq \hat{h}$. Then there exist $f_{0}, g_{0}, g_{1}$ and $h_{1}$ such that $f_{0} \in \hat{f}$, $g_{0} \in \hat{g}, g_{1} \in \hat{g}, h_{1} \in \hat{h}$ and such that $f_{0} \leqq g_{0}, g_{1} \leqq h_{1}$, a.e. If $g_{2}=g_{1}-g_{0}\left(\in \mathscr{C}^{\Phi}\right)$, then $f_{2}=f_{0}+g_{2} \in \hat{f}$ and $f_{2} \leqq g_{0}+g_{2}=g_{1} \leqq h_{1}$, a.e., so that $\hat{f} \leqq \hat{h}$. To see it is anti-symmetric, let $\hat{p}, \hat{q}$ in $\mathscr{N}^{\Phi}$ satisfy $\hat{p} \leqq \hat{q}$ and $\hat{q} \leqq \hat{p}$. Then there are $p_{0}, p_{1}$ in $\hat{p}, q_{0}, q_{1}$ in $\hat{q}$ such that $p_{0} \leqq q_{0}$, and $q_{1} \leqq p_{1}$, a.e. If $r=q_{1}-q_{0}\left(\in \mathscr{M}^{\Phi}\right)$, then $p_{2}=p_{0}+r \leqq q_{0}+r=q_{1} \leqq p_{1}$, a.e., so that $0 \leqq p_{1}-p_{2}=\left(p_{1}-p_{0}\right)-r \in \mathscr{C}^{\Phi}$ and $0 \leqq p_{1}-q_{1} \leqq p_{1}-p_{2} \in \mathscr{K}^{\Phi}$. So $p_{1}-q_{1} \in \mathscr{C l}^{\Phi}$, since $\mathscr{C}^{\Phi}$ is 'normal' (i.e., $g \in \mathscr{C l}^{\Phi},|h| \leqq|g|$, a.e. implies $h \in \mathscr{C l}^{\Phi}$ ). Thus both $p_{1}$ and $q_{1}$ belong to $\hat{p}$. Consequently $\hat{p}=\hat{q}$. It follows that ' $\leqq$ ' is a partial ordering in $\mathscr{N}^{\oplus}$. In a similar manner it can be easily checked that for $\hat{f}, \hat{g}$ in $\mathscr{N}^{\Phi}, f \in \hat{f}, g \in \hat{g}$, if $h=\sup (f, g)$ and $\hat{h}=h+\mathscr{C}^{\Phi}$, then $\hat{f} \leqq \hat{h}$ and $\hat{g} \leqq \hat{h}$ and $\hat{h} \in \mathscr{N}^{\oplus}$. It therefore follows that $\mathscr{N}^{\oplus}$ is also a vector lattice under this ordering with $\hat{h}=\sup (\hat{f}, \hat{g})=\hat{f} \vee \hat{g}$. Since under the norm (2.10), the map $\lambda$ is continuous (Lemma 3 ) it is trivial to note that the 'sup' and 'inf' (defined similarly and denoted $\vee$ and $\wedge$ ) operations in $\mathscr{N}^{\oplus}$ are continuous so that it is actually a Banach lattice. These and certain other lattice properties are summarized in the following.

LEMmA 5. The space $\mathscr{N}^{\Phi}$ with norm (2.10) is a Banach lattice under the ordering: For $\hat{f}, \hat{g}, \in \mathscr{N}^{\Phi}$ define $\hat{f} \leqq \hat{g}$ to mean $f \leqq g$ a.e., for some $f \in \hat{f}$ and $g \in \hat{g}$ and if $\hat{h}=h+\mathscr{C}^{\Phi}$ where $h=\max (f, g)$, then $\hat{h}=\hat{f} \vee \hat{g}$ is the 'sup' and similarly define the 'inf' as $\hat{k}=\hat{f} \wedge \hat{g}$. [Then $\left.\hat{h}, \hat{k} \in \mathscr{N}^{\Phi}.\right] \quad$ Also $\hat{f}, \hat{g} \in \lambda\left(\widetilde{L}^{\Phi}\right)$ implies $\hat{f} \wedge \hat{g}=\hat{k} \in \lambda\left(\widetilde{L}^{\varphi}\right)$ and $\hat{f} \vee \hat{g}=\hat{h} \in \lambda\left(\widetilde{L}^{\oplus}\right)$. Moreover, $\left(\mathscr{N}^{\oplus}\right)^{*}$, the adjoint space, is also a Banach lattice where the ordering is: For $z^{*} \in\left(\mathscr{N}^{\oplus}\right)^{*}$, let $z^{*} \geqq 0$ if and only if $z^{*}(\hat{f}) \geqq 0$ for all $\hat{f} \geqq \hat{0}$. Every $z^{*}$ in $\left(\mathscr{N}^{\Phi}\right)^{*}$ can be decomposed into the difference of two functionals as

$$
z^{*}=\left(z^{*}\right)^{+}-\left(z^{*}\right)^{-}, \quad\left(z^{*}\right)^{+} \wedge\left(z^{*}\right)^{-}=0,
$$

where $\left(z^{*}\right)^{+}$are positive functionals. ( $F$ is a positive functional if $F(f) \geqq 0$ whenever $f \geqq 0$.) 
Proof. The first part of the lemma, including the lattice property of $\lambda\left(\widetilde{L}^{\Phi}\right)$, is already proved in the preceding discussion, and this is just a restatement. So consider $\left(\mathscr{N}^{-\oplus}\right)^{*}$.

If $\hat{0} \leqq \hat{f} \leqq \hat{g}$ then by definition of norm (2.10), it follows that $d(\hat{f}) \leqq d(\hat{g})$. So if $z^{*} \in\left(\mathcal{N}^{\oplus}\right)^{*}$, then $\left|z^{*}(\hat{f})\right| \leqq\left\|z^{*}\right\| \cdot d(\hat{f}) \leqq\left\|z^{*}\right\| \cdot d(\hat{g})$, and this means that each $z^{*}$ in $\left(\mathscr{N}^{\oplus}\right)^{*}$ is bounded on every order interval, $\{\hat{f}: \hat{k} \leqq \hat{f} \leqq \hat{h}\}$ of $\mathscr{N}^{\Phi}$. Since $\mathscr{N}^{\Phi}$ is a vector lattice, it follows by $([3]$, p. $88,6(\mathrm{e}))$ that $\left(\mathscr{N}^{\oplus}\right)^{*}$ is also a vector lattice. By ([3], p. 99, Lemma 2) $\left(\mathscr{N}^{\oplus}\right)^{*}$ is a Banach lattice since $\mathscr{N}^{\oplus}$ is. Finally, (2.11) holds in every vector lattice $\left([22]\right.$, p. 391) and $\left(\mathscr{N}^{\Phi}\right)^{*}$ is just shown to be one. This completes the proof of the lemma.

Another important property of the elements of $\left(\mathscr{N}^{-\oplus}\right)^{*}$ is given in

Lemma 6. If $z^{*} \in\left(\mathscr{N}^{\Phi}\right)^{*}$ and $\left|z^{*}\right|=\left(z^{*}\right)^{+}+\left(z^{*}\right)^{-}$, where $\left(z^{*}\right)^{ \pm}$are the same symbols given in (2.11), then

$$
\begin{aligned}
\left\|z^{*}\right\| & =\left\|\left|z^{*}\right|\right\|=\sup \left\{\left|z^{*}\right|(\hat{f}): \hat{0} \leqq \hat{f} \in \lambda\left(\widetilde{L}^{\Phi}\right)\right\} \\
& =\left\|\left(z^{*}\right)^{+}\right\|+\left\|\left(z^{*}\right)^{-}\right\| .
\end{aligned}
$$

In fact, if $z_{1}^{*}$ and $z_{2}^{*}$ are any two positive elements of $\left(\mathscr{N}^{\oplus}\right)^{*}$, then

$$
\left\|z_{1}^{*}+z_{2}^{*}\right\|=\left\|z_{1}^{*}\right\|+\left\|z_{2}^{*}\right\| \text {. }
$$

Proof. The equality between the first three quantities of (2.12) holds in any Banach lattice on replacing $\lambda\left(\widetilde{L}^{\oplus}\right)$ by the unit ball there. Since the norm is determined by the elements of the closed or open unit ball in a $B$-space, and $\lambda\left(\widetilde{L}^{\varphi}\right)$ contains the open unit ball of $\mathscr{N}^{\Phi}$ and is contained in its closure (by Lemma 4 and Lemma 3(ii)) it follows that the norms of the elements of $\left(\mathscr{N}^{\oplus}\right)^{*}$ are determined by the elements of $\lambda\left(\tilde{L}^{\Phi}\right)$ alone. With this, the proof of all the equalities of (2.12) is similar to that given in ([22], p. 396), for the space of continuous functions with compact support, with simple modifications. The needed modifications will be indicated by proving (2.13) here.

Since $\left\|z_{1}^{*}+z_{2}^{*}\right\| \leqq\left\|z_{1}^{*}\right\|+\left\|z_{2}^{*}\right\|$ is always true for any $z_{1}^{*}, z_{2}^{*}$ in $\left(\mathscr{N}^{-\phi}\right)^{*}$, the hypothesis $z_{i}^{*} \geqq 0$ is needed only for the opposite inequality. The nontrivial case is when both $z_{1}^{*}, z_{2}^{*}$ are nonvanishing. Since, by Lemma $5, \hat{f}, \hat{g} \in \lambda\left(\tilde{L}^{\oplus}\right)$ implies $\hat{f} \vee \hat{g} \in \lambda\left(\widetilde{L}^{\phi}\right)$ and by the preceding paragraph $\left\|z_{i}^{*}\right\|=\sup \left\{z_{i}^{*}(\hat{f}): \hat{0} \leqq \hat{f} \in \lambda\left(\tilde{L}^{\phi}\right)\right)$, given $\varepsilon>0$, there exist $\hat{0} \leqq \hat{f}_{i} \in \lambda\left(\widetilde{L}^{\Phi}\right)$, such that $\left\|z_{i}^{*}\right\| \leqq z_{i}^{*}\left(\hat{f}_{i}\right)+\varepsilon / 2, i=1,2$. If $\hat{h}=\hat{f}_{1} \vee \hat{f}_{2}$, then $\hat{h} \in \lambda\left(\widetilde{L}^{\oplus}\right)$ and $\hat{f}_{i} \leqq \hat{h}$, and since $z_{i}^{*}$ is order-preserving, it follows that

$$
\left\|z_{1}^{*}\right\|+\left\|z_{2}^{*}\right\|-\varepsilon \leqq z_{1}^{*}\left(\widehat{f}_{1}\right)+z_{2}^{*}\left(\widehat{f}_{2}\right) \leqq\left(z_{1}^{*}+z_{2}^{*}\right)(\hat{h}) \leqq\left\|z_{1}^{*}+z_{2}^{*}\right\| .
$$


From the arbitrariness of $\varepsilon$, the desired inequality is obtained from the above. This proves (2.13), and the lemma follows as indicated.

REMARK. 1. The equation (2.13) implies $\left(\mathscr{N}^{-\oplus}\right)^{*}$ is an abstract $L$-space in the sense of Kakutani [10], and (even only with (2.12) $\left(\mathscr{N}^{\Phi}\right)^{*}$ is an abstract Banach lattice, in the sense of $([3]$, p. 98)). Hence, from the general properties of such lattices,

$$
\left\|z_{1}^{*}+z_{2}^{*}\right\|=\left\|z_{1}^{*}-z_{2}^{*}\right\| \text {, if }\left|z_{1}^{*}\right| \wedge\left|z_{2}^{*}\right|=0 \text {. }
$$

2. The results of Lemmas 5 and 6 and the discussion preceding Lemma 5, show that one can treat $\mathscr{N}^{\oplus}$ simply as a Banach function space with $\hat{f}$ in $\mathscr{N}^{\Phi}$ as a function to mean any member $f$ of $\hat{f}$. This is no different from considering $L^{\Phi}$ (or $L^{p}$ ) as a Banach function space by treating $f$ in $L^{\Phi}$ (or $L^{p}$ ) as a function to mean a member of its equivalence class. The ordering introduced in $\mathscr{N}^{\Phi}$ simply emphasizes this point. For this reason (when no confusion results), hereafter $\mathscr{N}^{\oplus}$ can be treated, and referred to, as a Banach function space. Thus, the decomposition (2.11) may also be obtained formally from the classical case (cf., [25], p. 192). Moreover $z^{*}$ in $\left(\mathscr{N}^{-\oplus}\right)^{*}$ is positive, with the ordering in $\mathscr{N}^{\Phi}$, if and only if $j^{-1}\left(z^{*}\right)$ in $\left(\mathscr{C}^{\Phi}\right)^{\perp}$ is positive in the usual order of $L^{\Phi}$. In fact, the order in $\mathscr{N}^{\Phi}$ was motivated by this fact and avoids the cumbersome references to $\left(\mathscr{l}^{\Phi}\right)^{\perp}$ at every stage. Here it may be mentioned that this point of view extends naturally for spaces of set functions and others as well. Mr. J. J. Uhl, Jr., has recently exploited this point successfully in his thesis [23], and proved several results on Orlicz spaces of finitely additive set functions.

3. Representation theorems. In this section various (integral) representations of continuous linear functionals on $L^{\Phi}$ spaces, where $\Phi$ is a Young's function will be presented, culminating in a complete characterization of $\left(L^{\Phi}\right)^{*}$ in Theorem 4 below. Hereafter all set functions will be assumed to have the (convenient) finite subset property, FSP, as discussed in some detail, for instance, in ([20], pp. 672-673).

It is convenient to call a Young's function $\Phi$, purely discontinuous if $\Phi(x)=0$ for $0 \leqq x \leqq x_{0}, x_{0}>0$, and $\Phi(x)>0$, for $x_{0} \leqq x \leqq x_{1},=\infty$ for $x>x_{1}$, and continuous in the extended sense (this was introduced in [17], p. 1452), if $\Phi(x)$ and $\Phi^{\prime}(x)$ are continuous for $0 \leqq x<x_{0}$, $\Phi^{\prime}(x)>0$ for $x>0, x_{0}>0$ and $\Phi(x) \rightarrow \infty$ and $\Phi^{\prime}(x) \rightarrow \infty$ as $x \rightarrow x_{0}$. Note that both these situations can occur for a discontinuous Young's function.

Definition 3. Let $\Phi$ be a Young's function and $A_{\Phi}(\mu)$ be the 
class of real additive set functions on $\Sigma$, vanishing on $\mu$-null sets, and satisfying the conditions: (i) (a) if the complementary function $\Psi$ of $\Phi$ is not purely discontinuous, then $G$ on $\Sigma_{1}$, the ring of sets of finite $\mu$-measure in $\Sigma$, is not identically zero and (b) if $\Psi$ is purely discontinuous, then $G$ is defined on all of $\Sigma$, (ii) $\|G\|_{\Phi}<\infty$ where

$$
\begin{aligned}
& \|G\|_{\Phi}=\inf \left\{k>0: I_{\Phi}\left(\frac{G}{k}\right) \leqq 1\right\}, \\
& I_{\Phi}(G, E)=\sup \sum_{i=1}^{n} \Phi\left(\frac{G\left(E_{i}\right)}{\mu\left(E_{i}\right)}\right) \mu\left(E_{i}\right),
\end{aligned}
$$

and where the supremum is taken relative to all finite disjoint collections of sets $\left\{E_{i}\right\} \subset \Sigma_{1} \cap E$, if $\Psi$ is not purely discontinuous and replace $\Sigma_{1}$ by $\Sigma$ otherwise in the last inclusion; and $I_{\mathscr{Q}}(G)=I_{\mathscr{Q}}(G, \Omega)$.

REMARK. It may be noted that if $\Psi$ is purely discontinuous with $0<x_{0}<x_{1}<\infty$ in its definition (i.e., $\Psi(x)=0$ for $0<x \leqq x_{0}, x_{0}>0$ for $x_{0} \leqq x \leqq x_{1}$, and $=\infty$ for $x>x_{1}$ ) then from the relationship between $\Phi, \Psi$ it follows that $x_{0}|x| \leqq \Phi(x) \leqq x_{1}|x|$, for all $x$ (see, e.g. [13], p. 11), and conversely. Thus in the above definition any reference to $\Psi$ may be suppressed, if so desired, using the fulfilment (or otherwise) of the above inequality. It should be remarked that in [15], [17], and [26] $\Phi^{\prime}$ was taken to be left continuous whereas in this paper and in [13] it is right continuous.

For the generalized Young's functions a slightly different formulation of the above definition is useful. [Compare with ([7], p. 592), where a similar concept was briefly discussed.] This will now be introduced and shown to be equivalent to the above definition for Young's functions.

Definition 4. Let $\Phi$ be a (generalized) Young's function and $\Psi$ its complementary function (cf. (2.1) or [26], or [15]). Let $A_{\phi}^{\prime}(\mu)$ be the class of real additive set functions $G$ on $\Sigma$, vanishing on $\mu$-null sets, such that $\|G\|_{\Phi}<\infty$ where

$$
\|G\|_{\Phi}=\sup \left\{\left|\int_{\Omega} f d G\right|:\|f\|_{\psi} \leqq 1\right\} \text {. }
$$

Here the integral relative to the finitely additive set function $G$ is understood as in ([6], Ch. III, or [2]), and $\|\cdot\|_{\psi}$ is defined in (2.3).

That (3.1) and (3.2) are norms and $A_{\varphi}(\mu)$ and $A_{\varphi}^{\prime}(\mu)$ are normed linear spaces is obvious. The relation between these spaces is given by the following.

Lemma 7. Let $\Phi$ be a Young's function. Then $A_{\varphi}(\mu)=A_{\Phi}^{\prime}(\mu)$ 
in the sense that they contain the same elements. Moreover, for any $G \in A_{\varphi}(\mu)$, if the complementary function $\Psi$ is not purely discontinuous, then

$$
\|G\|_{\Phi} \leqq\|G\|_{\Phi} \leqq 2\|G\|_{\Phi},
$$

and if $\Psi$ is purely discontinuous one has

$$
\alpha v(G) \leqq\|G\|_{\Phi} \leqq \beta\|G\|_{\Phi} \leqq \gamma v(G)
$$

where $\alpha, \beta$ and $\gamma$ are positive constants depending on $\Phi$ (or rather $x_{0}$ and $x_{1}$ ) and where $v(G)$ is the total variation (norm) of $G$.

Proof. It is sufficient to prove (3.3) and (3.4). Suppose first that $\Psi$ is not purely discontinuous. If $G \in A_{\phi}^{\prime}(\mu)$ is null then (3.3) is true and trivial so if $G \neq 0$, it may be assumed that $\|G\|_{\infty}=1$. Let $f \in L^{\psi}$ be a simple function such that $\|f\|_{\psi} \leqq 1, f=\sum_{i=1}^{n} a_{i} \chi_{E_{i}}$ where $0<\mu\left(E_{i}\right)<\infty, E_{i}$ disjoint, and $a_{i}=\Phi^{\prime}\left(G\left(E_{i}\right) / \mu\left(E_{i}\right)\right) \operatorname{sgn}\left(G\left(E_{i}\right)\right)$. This is possible since the $a_{i}$ may be replaced by $\alpha a_{i}$ in the representation of $f$ and $\alpha$ may be taken as $\left(\|f\|_{\psi}\right)^{-1}$ if $\|f\|_{\psi}>1$. Then one has

$$
\begin{aligned}
1 \geqq\left|\int_{0} f d G\right|= & \left|\sum_{i=1}^{n} a_{i}\left(\frac{G\left(E_{i}\right)}{\mu\left(E_{i}\right)}\right) \mu\left(E_{i}\right)\right| \\
= & \sum_{i=1}^{n} \Psi\left(a_{i}\right) \mu\left(E_{i}\right)+\sum_{i=1}^{n} \Phi\left(\frac{G\left(E_{i}\right)}{\mu\left(E_{i}\right)}\right) \mu\left(E_{i}\right) \\
& \text { by the condition for equality in (2.2), (cf. } \\
& {[26], \text { or }[15]), } \\
\geqq & \sum_{i=1}^{n} \Phi\left(\frac{G\left(E_{i}\right)}{\mu\left(E_{i}\right)}\right) \mu\left(E_{i}\right) .
\end{aligned}
$$

Since $\left\{E_{i}\right\} \subset \Sigma_{1}$ is arbitrary, it follows from the above that $I_{\phi}(G) \leqq 1$ and hence $\|G\|_{\Phi} \leqq 1=\|G\|_{\Phi}$ so that half of (3.3) is proved. For the second half, let $\|G\|_{\Phi}=1$, and $f=\sum_{i=1}^{n} b_{i} \chi_{E_{i}}$ be any other simple function such that (for convenience) $\|f\|_{\Psi} \leqq 1$. Then

$$
\begin{aligned}
\left|\int_{\Omega} f d G\right|= & \left|\sum_{i=1}^{n} b_{i}\left(\frac{G\left(E_{i}\right)}{\mu\left(E_{i}\right)}\right) \mu\left(E_{i}\right)\right| \\
\leqq & \sum_{i=1}^{n} \Psi\left(b_{i}\right) \mu\left(E_{i}\right)+\sum_{i=1}^{n} \Phi\left(\frac{G\left(E_{i}\right)}{\mu\left(E_{i}\right)}\right) \mu\left(E_{i}\right) \\
& \text { by Young's inequality }(2.2), \\
& \int_{\Omega} \Psi(f) d \mu+I_{\oplus}(G) \leqq 2
\end{aligned}
$$

Since $f$ is arbitrary, this implies $\|G\|_{\Phi} \leqq 2=2\|G\|_{\odot}$. This completes the proof of (3.3). 
For (3.4), let $\Psi$ be purely discontinuous. Then by the remark following Definition 3, and (3.1), one has

$$
x_{0} v(G)=\sup \left[x_{0} \sum_{i=1}^{n}\left|G\left(E_{i}\right)\right|\right] \leqq I_{\Phi}(G) \leqq \sup x_{1}\left[\sum_{i=1}^{n}\left|G\left(E_{i}\right)\right|\right]=x_{1} v(G) .
$$

Hence $x_{0} v(G) \leqq\|G\|_{\odot} \leqq x_{1} v(G)$, i.e., $\|\cdot\|_{\Phi}$ and the variation norm are equivalent. On the other hand, as shown in the proof of Lemma 2, in the present case $L^{\psi}=L^{\infty}=\mathscr{C l}^{\Psi}$ and since $\|f\|_{\infty} \leqq x_{1} N_{\psi}(f) \leqq x_{1}\|f\|_{\psi}$, the norms are equivalent. So there is a positive constant $\alpha_{0}$ (depending only on $\Psi$ and hence only on $\Phi$ ) such that $\alpha_{0}\|f\|_{\psi} \leqq\|f\|_{\infty} \leqq x_{1}\|f\|_{\psi}$. Since $\sup \left\{\left|\int_{0} f d G\right|:\|f\|_{\infty} \leqq 1\right\}=v(G)$ (cf. [6], p. 296) it follows that $\alpha_{0}\|G\|_{\Phi} \leqq v(G) \leqq x_{1}\|G\|_{\psi}$, i.e., $\|\cdot \cdot\|_{\phi}$ and the variation norm are equivalent. Thus this and the earlier inequality imply (3.4) at once, for some appropriate (positive) constants $\alpha, \beta$ and $\gamma$, completing the proof.

REMARK. In view of the above lemma hereafter the prime on $A_{\Phi}^{\prime}(\mu)$ can and will be dropped. Also if $x_{0}=x_{1}=1$ then it can be seen that, in (3.4), $\alpha=\beta=\gamma=1$ and there is equality throughout.

The inequalities (3.3) may be refined in many cases. This can be seen after proving the following

LEMma 8. If $\Phi$ is a Young's function such that its complementary function $\Psi$ is continuous, then every member of $A_{\mathscr{\Phi}}(\mu)$ is countably additive.

Proof. When $\Psi$ is continuous, by ([19], Lemma 6) every $G \in A_{\odot}(\mu)$ is $\mu$-continuous, i.e., $|G(E)| \rightarrow 0$ as $\mu(E) \rightarrow 0$. Hence if $\left\{E_{n}\right\} \subset \Sigma_{1}$ and the $E_{n}$ decrease to $\varnothing$, the empty set, then $\left|G\left(E_{n}\right)\right| \rightarrow 0$ so that $G(\cdot)$ is continuous at $\varnothing$. This implies in turn that $G$ is countably additive on $\Sigma_{1}$. So by standard extension procedures of measure theory (cf. [9], p. 54), $G$ is countably additive on the $\sigma$-ring $S\left(\Sigma_{1}\right)$ generated by $\Sigma_{1}$. It remains to show that there exists a set $E$ in $S\left(\Sigma_{1}\right)$ such that $G$ coincides with its restriction $G_{E}$ to $E$, (i.e., $G_{E}=G(E \cap \cdot)$ ). This is seen as follows.

From the definition of $I_{\Phi}(G, A)$ in (3.1), it is not hard to see that $I_{\phi}(G, \cdot)$ is an additive set function on $\Sigma_{1}$. (Details of computations of this remark were given by Uhl [23].) It is clear that $I_{\phi}(G, A)=0$ if $\mu(A)=0$. Since the complementary function $\Psi$ of $\Phi$ is continuous by hypothesis, it follows again by $\left([19]\right.$, Lemma 6) that $I_{\odot}(G, \cdot)$ is $\mu$-continuous. But then as in the preceding paragraph $I_{\mathscr{D}}(G, \cdot)$ must be countably additive on $S\left(\Sigma_{1}\right)$ and by hypothesis $I_{\bullet}(G, \Omega)<\infty$. Hence by definition in $(3.1)$, since $I_{\phi}(G, \cdot)$ is a measure, there exists a sequence 
$\left\{A_{n}\right\} \subset \Sigma_{1}, A_{n} \subset A_{n+1}$, such that $\lim _{n \rightarrow \infty} I_{\phi}\left(G, A_{n}\right)=I_{\phi}(G)$. If $E=\bigcup_{n=1}^{\infty} A_{n}$, then it follows easily that $I_{\mathscr{D}}(G, E)=I_{\mathscr{Q}}(G)<\infty, E \in S\left(\Sigma_{1}\right)$, and $I_{\varphi}\left(G, E^{\prime}\right)=0$ where $E^{\prime}=\Omega-E$ (cf. also [19], p. 80). This means $G=G_{E}$, as was to be proved. [An alternate proof can be given using ([16], Theorem 7.1).]

In view of the preceding lemma the following result is of interest as it improves upon (3.3).

CoRollary. If $G \in A_{\Phi}(\mu)$ is countably additive (in particular if the complementary function $\Psi$ of $\Phi$ is continuous) then

$$
\|G\|_{\Phi}=\|G\|_{\Phi} \text {. }
$$

Proof. If $G$ is countably additive, and since it vanishes on $\mu$-null sets, it follows that, by a generalized Radon-Nikodým theorem (cf. [16], p. 336), there exists a quasi-function $g^{*}$ (i.e., one which is consistently equal to a measurable function on sets of finite $\mu$ measure) such that $G(E)=\int_{E} g^{*} d \mu, E \in \Sigma$, and then by ([19], Lemma 5) $I_{\phi}(G)=\int_{\Omega} \Phi\left(g^{*}\right) d \mu$. (Integrals are defined for quasi-functions, as pointed out in [16].) This and (3.3) imply (see also (2.4)) immediately $\|G\|_{\phi}=N_{\Phi}\left(g^{*}\right)$. But simple functions in $L^{\Phi}$ are norm-determining so that

$$
\begin{aligned}
\|G\|_{\Phi}=N_{\varphi}\left(g^{*}\right) & =\sup \left\{\left|\int_{\Omega} g^{*} f d \mu\right|:\|f\|_{\psi} \leqq 1, f \text { simple }\right\} \\
& =\sup \left\{\left|\int_{\Omega} f d G\right|:\|f\|_{\psi} \leqq 1\right\}=\|G \mid\|_{\varnothing} .
\end{aligned}
$$

This completes the proof of (3.5).

In terms of the work of this paper, the main result of [19] will be restated more precisely as follows. Hereafter, unless the contrary is stated, the norm in $L^{\Phi}$ is that given by (2.3) and that in $L^{\Psi}(\Psi$ complementary to $\Phi$ ) is that qiven by (2.4). Also $\Phi$ stands for $a$ Young's function.

Theorem 1. For each $x^{*} \in\left(\mathscr{C}^{\Phi}\right)^{*}$, the adjoint space of $\mathscr{L}^{\Phi}$, there exists a unique $G \in A_{w}(\mu)$, such that

$$
x^{*}(f)=\int_{\Omega} f d G, \quad f \in \mathscr{C}^{\Phi}
$$

and

$$
\left\|x^{*}\right\|=\|G\| \|_{\psi},
$$

so that $\left\|x^{*}\right\|=\|G\|_{\Psi}$ if $\Phi$ is continuous and $\alpha\left\|x^{*}\right\| \leqq\|G\|_{\psi} \leqq \beta\left\|x^{*}\right\|$ for some positive $\alpha, \beta$, depending only on $\Phi$, otherwise. 
Proof. Since the proof of this result is essentially given in [19], so only an outline (based on the preceding lemmas) will be presented here for completeness. If $G \in A_{\Psi}(\mu)$, and $\Phi$ is continuous, then, as in the proof of the above Corollary, it follows that

$$
x^{*}(f)=\int_{\Omega} f d G=\int_{\Omega} f g^{*} d \mu, \quad f \in \mathscr{L}^{\bullet} .
$$

By means of Hölder's inequality, the above equation defines a bounded linear functional and, by (3.5), $\left\|x^{*}\right\|=\|G\|_{\psi}=\|\| G \|_{\psi}$. If $\Phi$ is discontinuous, then for each $E \in \Sigma_{1}$, and $G \in A_{w}(\mu)$, (3.6) is well defined for $f \in \mathscr{C}^{\Phi}(E)=L^{\Phi}(E)=L^{\infty}(E)$ where the latter symbols stand for the spaces restricted to $E$. Moreover, (cf. [6], p. 296),

$$
\left|\lambda_{G}(E)\right|=\left|\int_{E} f d G\right| \leqq\|f\|_{\infty} v\left(G_{E}\right) \leqq k\|f\|_{\odot}\|G\|_{\Psi},
$$

by Lemma 7 . Here $k$ depends only on $\Phi$, and $G_{E}$ is the restriction of $G$ to $E$. But $\lambda_{0}(\cdot)$ on $\Sigma_{1}$ is a finitely additive bounded set function. So by ([6], IV. 9.11) $\lambda_{0}$ can be isometrically mapped onto a regular countably additive bounded (by $k\|f\|_{\Phi}\|G\|_{\psi}=\alpha$ ) set function $\tilde{\lambda}_{0}$ which satisfies the inequality $\left|\widetilde{\lambda}_{0}\left(S_{1}\right)\right| \leqq \alpha<\infty$, where $S_{1}$ is the image of $\Omega$ under the map. Using the isometry again one concludes that $\left|\lambda_{0}(\Omega)\right| \leqq \alpha<\infty$, so that (3.6) defines a bounded linear functional on not only $\mathscr{C}^{\oplus}$, but on $L^{\oplus}$ itself. (The details of the compressed argument here may also be found in [19], pp. 88-89.) Then (3.7) follows from the definition of the norms involved. The last statement now is a consequence of Lemmas 7 and 8.

Conversely if $x^{*} \in\left(\mathscr{C}^{\oplus}\right)^{*}$, then $G(E)=x^{*}\left(\chi_{E}\right), \chi_{E} \in \mathscr{C l}^{\oplus}$, shows that $G$ is an additive real set function vanishing on $\mu$-null sets, and (3.6) holds for all simple $f$ in $\mathscr{C}^{\Phi}$. If $\Phi$ is purely discontinuous then $|G(E)| \leqq\left\|x^{*}\right\|\left\|\chi_{E}\right\|_{\phi} \leqq k\left\|x^{*}\right\|\left\|\chi_{\Omega}\right\|_{\infty}=k\left\|x^{*}\right\|<\infty$ for some $k>0$, depending only on $\Phi$, and where $L^{\infty}=L^{\Phi}=\mathscr{C}^{\Phi}$ is used. But then, by ([6], p. 97), $v(G) \leqq k\left\|x^{*}\right\|$ so that by (3.4), $G \in A_{\Psi}(\mu)$ and, as in the first paragraph, (3.6) and (3.7) hold in this case. If $\Phi$ is not purely discontinuous, then a procedure similar to that in the proof of Lemma 7 can be applied. Thus, excluding the true and trivial case $x^{*}=0$, let $f=\sum_{i=1}^{n} a_{i} \chi_{E_{i}}, 0<\mu\left(E_{i}\right)<\infty$, where

$$
a_{i}=\Phi^{\prime}\left(\frac{G\left(E_{i}\right)}{\left\|x^{*}\right\| \mu\left(E_{i}\right)}\right) .
$$

Since $f$ vanishes outside of a $\mu$-finite set, it follows that

$$
\|f\|_{\Phi} \leqq \int_{\Omega} \Phi(f) d \mu+1<\infty,
$$

(by Young's inequality and definition (2.3)) and one has 


$$
\begin{aligned}
1+\int_{\Omega} \Phi(f) d \mu \geqq \frac{x^{*}(f)}{\left\|x^{*}\right\|} & =\sum_{i=1}^{n} a_{i}\left(\frac{G\left(E_{i}\right)}{\left\|x^{*}\right\| \mu\left(E_{i}\right)}\right) \mu\left(E_{i}\right) \\
& =\int_{\Omega} \Phi(f) d \mu+\sum_{i=1}^{n} \Psi\left(\frac{G\left(E_{i}\right)}{\left\|x^{*}\right\| \mu\left(E_{i}\right)}\right) \mu\left(E_{i}\right)
\end{aligned}
$$

by equality in (2.2).

This implies, since $\left\{E_{i}\right\} \subset \Sigma_{1}$ is arbitrary, $\|G\|_{x} \leqq\left\|x^{*}\right\|<\infty$. So $G \in A_{w}(\mu)$, and by the result of the first paragraph, (3.6) and (3.7) hold for this $G$. Uniqueness being evident, the essential details of the proof of the theorem are completed.

REMARK. The last part is again the same as in [19]. But it should be noted that the employment of the two (equivalent) norms in (3.1) and (3.2) makes the proof rather transparant in contrast to using only (3.1) all the time.

As a consequence of the above result one has the following.

Proposition 1. Let $\Phi$ be a Young's function and $\Psi$ its complementary function. Then $L^{\Phi}$ is reflexive if and only if $L^{\Phi}=M^{\Phi}$ and $L^{\psi}=M^{\psi}$, where $M^{\Phi},\left(M^{\psi}\right)$ is defined before Lemma 2 .

Proof. If $L^{\Phi}=M^{\Phi}$ and $L^{\Psi}=M^{\psi}$, then this implies $\tilde{L}^{\Phi}=M^{\Phi}$ and $\widetilde{L}^{\Psi}=M^{\Psi}$ at once. In this case the result is immediate from Theorem 1 , and was also given in ([19], Th. 5, cf. also [20], Theorem 4). Conversely if $L^{\Phi}$ is reflexive then, $L^{\Phi} \subset\left(L^{\psi}\right)^{*} \subset\left(L^{\Phi}\right)^{* *}=L^{\Phi}$ so that $\left(L^{\psi}\right)^{*}=L^{\Phi}$ and, by a well known result of Pettis (cf. [6], p. 67), $L^{\psi}$ is reflexive. [Here, as usual, the inclusions and equalities are in terms of the natural imbeddings.] Hence $\left(L^{\Phi}\right)^{*}=\left(L^{\Psi}\right)^{* *}=L^{\psi}$. However, this holds if and only if the norms in $L^{\Phi}$ and $L^{\psi}$ are absolutely continuous, i.e., the condition given in ([8], p. 205). This means $M^{\oplus} \subset L^{\Phi} \subset M^{\Phi}$, and a similar statement holds for $L^{\psi}$. Consequently the condition of the Proposition is necessary, completing the proof.

REMARK. If the measure space is $\sigma$-finite, a similar result was given in ([15], p. 60). (cf. also $\S 4$ below for some related results.)

The above Theorem 1 does not give information about functionals in $\left(\mathscr{C l}^{\oplus}\right)^{\perp}$, or equivalently $\left(\mathscr{N}^{\bullet}\right)^{*}$ in the notation of Lemma 3. Using the work of $\S 2$ above, integral representations of the elements of $\left(\mathscr{N}^{\Phi}\right)^{*}$ can now be given. It will be shown, in Theorem 4 below, that the general $x^{*}$ in $\left(L^{\Phi}\right)^{*}$ may be given an integral representation, as in (3.6), with an extension of the definition of the integral.

It was pointed out after Lemma 6 above that $\left(\mathscr{N}^{\oplus}\right)^{*}$ is an abstract $(L)$-space [10]. A characterization of abstract $(L)$-spaces, 
which are Banach lattices (so $\left(2.13^{\prime}\right)$ also holds), was obtained by Kakutani in [10]. This result will be quoted here as it enables a better understanding of the structure of $\left(\mathscr{N}^{\oplus}\right)^{*}$.

THEOREM 2. (Kakutani) An abstract (L)-space $\mathscr{Z}$ satisfying $\left(2.13^{\prime}\right)$ and having a unit (i.e., there is an $x_{0} \in \mathscr{X}$ such that for every $0<x \in \mathscr{X}, x_{0} \wedge x>0$ holds), is isometric and lattice isomorphic to the Lebesgue space of integrable functions, $L^{1}(S, \mathscr{A}, \alpha)$, on some $(S, \mathscr{A}, \alpha)$ where $S$ is a (totally disconnected) compact topological space, $\mathscr{A}$ is the Borel field of $S$ and $\alpha$ is a (countably additive) measure on $\mathscr{A}$.

Even though $\left(\mathscr{N}^{\Phi}\right)^{*}$ satisfies $(2.13)$ and $\left(2.13^{\prime}\right)$, it does not have a unit in the sense of the above theorem. [Recall that $\mathscr{N}^{\Phi} \neq\{0\}$ so that $\mu$ is a nontrivial measure and since $\Phi$ does not satisfy any growth condition $\left(L^{\Phi}\right.$ and) $\mathscr{N}^{\Phi}$ and hence $\left(\mathscr{N}^{\oplus}\right)^{*}$ are nonseparable which follows, for instance, from $\left([15]\right.$, p. 61) also, so that $L^{1}(S, \mathscr{A}, \alpha)$ is not separable. Therefore, it cannot be the conjugate space of any $B$-space. Thus $\left(\mathscr{N}^{-\oplus}\right)^{*}$ and $L^{1}(S, \mathscr{A}, \alpha)$ cannot be isometric and lattice isomorphic which means there cannot be a unit in $\left(\mathscr{N}^{-}\right)^{*}$.] On the other hand, the result of Lemma 6 is well-known if $\mathscr{N}^{\Phi}$ is replaced by $L^{\infty}$. This 'suggests' that the abstract $(L)$-space $\left(\mathscr{N}^{-\oplus}\right)^{*}$ is equivalent to some subspace of $b a(\Omega, \Sigma, \mu)$, the $B$-space of bounded additive set functions on $\Sigma$ and vanishing on $\mu$-null sets with total variation as norm. This is indeed the case and the first step in this direction can be obtained as follows.

If $\hat{f} \in \mathscr{N}^{\Phi}$, let $\hat{f}_{E}$ stand for the coset $f_{E}+\mathscr{N}^{\Phi}$, and if $z^{*} \in\left(\mathscr{N}^{\Phi}\right)^{*}$, let $z_{E}^{*}$ be defined by $z_{E}^{*}(\hat{f})=z^{*}\left(\hat{f}_{E}\right), \hat{f} \in \hat{N}^{\oplus}$. With this notation one has

Lemma 9. To each $z^{*} \in\left(\mathscr{N}^{\cdot \oplus}\right)^{*}$ corresponds $a \nu \in b a(\Omega, \Sigma, \mu)$ which is purely finitely additive if $\nu \not \equiv 0$ (in the sense of [6], p. 163), and an $\hat{f} \in \mathscr{N}^{\Phi}, d(\hat{f})=1$, such that $z_{(\cdot)}^{*}$ and $\nu(\cdot)$ are related by the equations

$$
z_{E}^{*}(\widehat{f})=z^{*}\left(\hat{f}_{E}\right)=\nu(E), \quad E \in \Sigma
$$

and

$$
\left\|z_{E}^{*}\right\|=|\nu|(E)
$$

where $|\nu|(\cdot)$ is the variation measure of $\nu$.

Proof. In view of (2.12) it suffices to consider $z^{*} \geqq 0$ for this proof. If $E, F \in \Sigma$ are disjoint and $E \cup F=\Omega$, then (2.13) implies 


$$
\left\|z^{*}\right\|=\left\|z_{E \cup F}^{*}\right\|=\left\|z_{E}^{*}\right\|+\left\|z_{F}^{*}\right\|,
$$

so that $\left\|z_{(\cdot)}^{*}\right\|=\nu(\cdot)$ defines a positive bounded additive set function which vanishes on $\mu$-null sets. Thus (3.9) holds.

That $\nu(\cdot)$ is purely finitely additive (if $\nu \neq \equiv 0$ ), can be proved thus. For any $\varepsilon>0$, there exists $\hat{0} \leqq \hat{f}_{0} \in \mathscr{N}^{\oplus}$, depending on $\varepsilon$, such that $z^{*}\left(\hat{f}_{0}\right)=\left\|z^{*}\right\|$ and $\left|d\left(\hat{f}_{0}\right)-1\right|<\varepsilon$. (This is a simple consequence of the definition of norm, and it holds in any $B$-space.) If $\tilde{\nu}(E)=$ $z^{*}\left(\hat{f}_{0 E}\right)=z_{E}^{*}\left(\hat{f}_{0}\right), E \in \Sigma$, then $0 \leqq \tilde{\nu} \in b a(\Omega, \Sigma, \mu)$, and $\nu(\Omega)=\tilde{\nu}(\Omega)$. Moreover $\tilde{\nu}(E) \leqq \nu(E)(1+\varepsilon)$, for $E \in \Sigma$ so that $|\nu-\tilde{\nu}|(\Omega)<\varepsilon$. But there is a decreasing sequence $E_{n}$ in $\Sigma$ such that $\mu\left(E_{n}\right) \rightarrow 0$ and $\tilde{\nu}\left(E_{n}\right)=\widetilde{\nu}(\Omega)$ for all $n$ and this means $\widetilde{\nu}$ is purely finitely additive. In fact if $f_{0}=\lambda^{-1}\left(\hat{f}_{0}\right)$ where $\lambda: L^{\Phi} \rightarrow \mathscr{N}^{-\Phi}$ is the canonical map, and if $E_{n}=$ $\left\{t: f_{0}(t) \geqq n\right\} \in \Sigma$, then the $E_{n}$ sequence satisfies the above requirement since $f_{0}-f_{0 E_{n}} \in \mathscr{C l}^{\Phi}$ so that $z^{*}\left(\hat{f}_{0}\right)=z^{*}\left(\hat{f}_{0 E_{n}}\right)$. Now recalling the fact that the set of purely finitely additive set functions in $b a(\Omega, \Sigma, \mu)$ is a closed linear subspace, it follows from the inequality $|\nu-\widetilde{\nu}|(\Omega)<\varepsilon$ that $\nu$ is also purely finitely additive.

The proof of (3.8) will be postponed until the end of the proof of Theorem 3 below.

REMARKS. $\nu(\cdot), \widetilde{\nu}(\cdot)$ and $\hat{f}_{0}$ of the above proof (and also $\hat{f}$ of (3.8)) have the following noteworthy properties, (for convenience $f$ will be written for $f_{0}$ and $\nu$ for $\widetilde{\nu}$; and this will be exact when (3.8) is established):

(a) If $f=\lambda^{-1}(\hat{f}), E=\{t:|f(t)| \leqq x\}$, and $\nu_{E}$ is the restriction of $\nu$ to $E$ then $\nu_{E}(F)=z^{*}\left(\hat{f}_{E \cap F}\right)=0$ for every $0 \leqq x<\infty$ and $F \in \Sigma_{1}$ since $f_{E \cap F} \in \mathbb{K}^{\Phi}$.

(b) Since $\mathscr{N}^{\oplus}$ is nontrivial only if $\Phi(x)>0$ for $0<x \leqq a$ for some $a$, it follows from the integrability of $\Phi(f / k)$, for some $k>0$, that $\Omega-E$ has finite $\mu$-measure for every $x$ and if $\Phi$ is continuous then this measure tends to zero as $x \rightarrow \infty$.

(c) If $f$ is as in (a), then $f / k \in \widetilde{L}^{\Phi}$ for some $k \geqq 1$ (since $f \in L^{\Phi}$ and $d(\hat{f}) \geqq 1$ ), and by the structure theorem for measurable functions (cf. [9] p. 85), there exists a sequence of step functions $f_{n}$, all of whose supports lie in the support of $f$, such that $f_{n} \rightarrow f$ everywhere. Moreover the sequence can be chosen such that $\left|f-f_{n}\right| \leqq|f|$ and that the positive (negative) part of $f$ is approximated by the positive (negative) part of $f_{n}$ monotonously. Thus $f_{n} \in L^{\Phi}$. If $\mathscr{N}^{\Phi}$ is nontrivial, $\Phi$ cannot be purely discontinuous, and hence every step function in $L^{\Phi}$ is also a simple function, as pointed out in the remark after Lemma 2. It follows that $f_{n} \in \mathscr{C}^{\Phi}$ for every $n$ and by the dominated convergence theorem, for large enough $n, \int_{\Omega} \Phi\left(\left(f-f_{n}\right) / k\right) d \mu$ can be made arbitrarily small, i.e., for any $\varepsilon>0$ there is a $g \in \mathscr{C l}^{\Phi}$ such that 
$\int_{\Omega} \Phi((f-g) / k) d \mu<\varepsilon$. If $h=(f-g) / k$, then $h \in \widetilde{L}^{\Phi}$ with the important property: the support of $\nu$ (i.e., the sets $E$ for which $|\nu|(E)>0$ ) lies in the support of $h \in \widetilde{L}^{\Phi}-\mathscr{C}^{\Phi}$ (set theoretic difference). If $\mu(\Omega)<\infty$, then the support of $h$ itself has arbitrarily small $\mu$-measure. This last observation is useful in the analysis of the structure of singular linear functionals on general Banach function spaces, ([12], [14], [15]).

It may be noted that, from Lemma 6 , every element $x^{*}$ of the abstract $(L)$-space $\left(\mathscr{N}^{-\phi}\right)^{*}$ can be given an integral representation of the abstract Radon-Stieltjes type (cf. [10], Th. 6). However, in such a representation the particular $\mathscr{N}^{\oplus}$ itself is ignored, and consequently a different procedure is necessary. For this, first $\mathscr{N}^{\Phi}$ will be mapped isometrically into a subspace of $L^{\infty}$ and then an integral (see below) of such functions relative to the measure $\nu(\cdot)$ of Lemma 9 can be defined. The idea here is to 'associate' a more convenient abstract (M)-space with $\mathscr{N}^{\Phi}$ so that by ([11], Th. 15) the abstract $(L)$-space $\left(\mathscr{N}^{\Phi}\right)^{*}$ is determined. This construction will now be carried out.

Let $\hat{0} \leqq \hat{f} \in \mathscr{N}^{\Phi}$ and $\beta=d(\hat{f})$ where $d(\cdot)$ is given by $(2.10)$. Select $f \in \hat{f}$. Since by definition of ' $\leqq$ ' in $\mathscr{N}^{\oplus}$ there exists a $g \in \mathscr{C l}^{\mathscr{\Phi}}$ with $f-g \geqq 0$, a.e., (and $f-g \in \hat{f}$ ) it can be assumed that $f>0$, a.e. since $\beta>0$ is the only nontrivial case. Let $\pi=\left(E_{1}, \cdots, E_{n}\right)$ be a partition of $\Omega$ (i.e. a finite disjoint collection of sets in $\Sigma$ whose union is $\Omega)$. Set $f_{E}=f \chi_{E}$, as before and let $a_{k}=d\left(\hat{f}_{E_{k}}\right) \leqq \beta$. If $\nu \in b a(\Omega, \Sigma, \mu)$, consider the sum $\sum_{i=1}^{n} a_{i} \nu\left(E_{i}\right)$. As the partitions are refined it is seen that these sums form a (bounded) monotone sequence of numbers and they converge to a limit which is denoted by $\int_{\Omega} f d \nu$. If $\widetilde{f}_{\pi}=\sum_{i=1}^{n} a_{i} \chi_{E_{i}}$ relative to a partition $\pi$, then $\tilde{f}_{\pi} \in L^{\infty}$ and $\tilde{f}_{\pi}=0$ if and only if $f \in \mathbb{C}^{\Phi}$. Moreover, $\left\|\widetilde{f}_{\pi}\right\|_{\infty}=\max \left(a_{i}\right)=d(\hat{f})$, so that the map $\hat{f} \rightarrow \widetilde{f}_{\pi}$ is an isometry of $\mathscr{N}^{\oplus \Phi}$ onto a subspace of $L^{\infty}$. Let $x^{*}$ be the functional defined by

$$
x^{*}(\hat{f})=\int_{\Omega} f d \nu, \quad \hat{0} \leqq \hat{f} \in \mathscr{N}^{\Phi}, 0 \leqq f \in \hat{f} .
$$

Then one can show (with arguments similar to, e.g., [21], Chapitre VI; [22], p. 405) that $x^{*}$ is well-defined and is linear. This is clear in $\nu$, but the linearity in $\hat{f}$ needs some computation. In fact the following is true.

LEMMA 10. The functional $x^{*}$, given by (3.11), satisfies: $x^{*} \in\left(\mathscr{N}^{\Phi}\right)^{*}$, and (ii) $0 \leqq x^{*}(\hat{f}) \leqq d(\hat{f}) \nu(\Omega), \hat{0} \leqq \hat{f} \in \mathscr{N}^{\Phi}$.

Proof. (ii) follows directly from the definition of (3.11). Since $x^{*}(a \hat{f})=a x^{*}(\hat{f})$ for $a \geqq 0$, (i) follows from (ii) if the additivity of $x^{*}$ 
is established. Also since $d(\cdot)$ is a lattice norm, it is immediate from the definition that $x^{*}\left(\hat{f}_{1}+\hat{f}_{2}\right) \leqq x^{*}\left(\hat{f}_{1}\right)+x^{*}\left(\hat{f}_{2}\right)$ for any $\hat{0} \leqq \hat{f}_{1}, \hat{f}_{2}$ in $\mathscr{N}^{\Phi}$. For the opposite inequality let $0 \leqq f_{i} \in \hat{f}_{i}$. The result is successively deduced as follows.

If $f_{1}$ and $f_{2}$ have disjoint supports (letting $f=f_{1}+f_{2}$ in the definition of $d(\cdot)$ and the integral) it is seen that $x^{*}\left(\hat{f}_{1}+\hat{f}_{2}\right)=$ $x^{*}\left(\hat{f}_{1}\right)+x^{*}\left(\hat{f}_{2}\right)$. If $\hat{f}_{1}, \hat{f}_{2}$ are such that $0<f_{1} \leqq f_{2}$ then, since $x\left(f_{1}\right) \leqq$ $x^{*}\left(f_{2}\right)$ and $f_{1} / f_{2} \in L^{\infty}$, one proceeds as follows. The computation may be found in ([21], [1]). The procedure of ([21], p. 187) gives the additivity on using the existence of the limit in (3.11). Following [1], an alternate procedure can and will be given because of its interest. Even here, however, the existence of the limit in (3.11) will be implicitly used.

Since the bounded function $f_{1} / f_{2}$ can be approximated by step functions (cf. [6], p.296), for any $\varepsilon>0$, let $f_{\varepsilon}=\sum_{i=1}^{n} \alpha_{i} \chi_{E_{i}}, \alpha_{i} \geqq 0$, and $E_{i}$ disjoint in $\Sigma$, such that $0 \leqq\left(f_{1} / f_{2}\right)-f_{\varepsilon} \leqq \varepsilon / k$, a.e., where $0<k=x^{*}\left(\hat{f}_{2}\right)$. This may be rearranged, on letting $\alpha_{0}=0$ and $E_{0}=$ $\Omega-\bigcup_{i=1}^{n} E_{i}$, as

$$
\sum_{i=0}^{n} \alpha_{i} f_{2} \chi_{E_{i}} \leqq f_{1} \leqq \sum_{i=0}^{n} \alpha_{\imath} f_{2} \chi_{E_{i}}+f_{2} \varepsilon / k
$$

The monotonicity and additivity of $x^{*}$ for 'functions' of disjoint support (on noting the distributivity of the ' $\wedge$ ' notation) give,

$$
\begin{aligned}
x^{*}\left(\hat{f}_{1}\right)+x^{*}\left(\hat{f}_{2}\right) & \leqq \sum_{i=0}^{n} x^{*}\left(\widehat{f_{2}} \chi_{E_{i}}\right)+\sum_{i=0}^{n}\left(\alpha_{i}+\frac{\varepsilon}{k}\right) x^{*}\left(\widehat{f}_{2} \chi_{E_{i}}\right) \\
& =\sum_{i=0}^{n}\left(1+\alpha_{i}\right) x^{*}\left(\widehat{f_{2}} \chi_{E_{2}}\right)+\frac{\varepsilon}{k} x^{*}\left(\hat{f}_{2}\right) \\
& =x^{*}\left(\sum_{i=0}^{n}\left(1+\alpha_{i}\right) \widehat{f}_{2} \chi_{E_{i}}\right)+\varepsilon \leqq x^{*}\left(\hat{f}_{1}+\hat{f}_{2}\right)+\varepsilon .
\end{aligned}
$$

Hence $x^{*}\left(\hat{f}_{1}\right)+x^{*}\left(\hat{f}_{2}\right)=x^{*}\left(\hat{f}_{1}+\hat{f}_{2}\right)$ for $\hat{0}<\hat{f}_{1} \leqq \hat{f}_{2}$. If $\hat{f}_{1}, \hat{f}_{2} \geqq \hat{0}$ so that $0 \leqq f_{i} \in \hat{f}_{i}$ are arbitrary, one extends the above case by splitting $\Omega$ into $\left\{f_{1} \leqq f_{2}\right\}$ and its complement and then applying the preceding case. This completes the proof of (i) and of the lemma.

REMARK. Since by (2.11) every element of $\left(\mathscr{N}^{\Phi}\right)^{*}$ can be decomposed into its positive and negative parts, and a similar result holds for the elements of $\mathscr{V}^{\oplus}$ which is a vector lattice, (3.11) can be extended to all elements of $\left(\mathscr{N}^{\oplus}\right)^{*}$ and all $\hat{f}$ of $\mathscr{N}^{\oplus}$ by taking an appropriate linear combination (of four terms) of the integrals in the usual manner. This general case (integral) will be denoted by the same symbol as in (3.11).

Motivated by the result of Lemma 9 and following remarks, the 
definition below is introduced.

DEFINITION 5. Let $B_{\psi}(\mu) \subset b a(\Omega, \Sigma, \mu)$ be the class of set functions $\nu$, whose support lies in the support of some $f \in \widetilde{L}^{\Phi}-\mathscr{C}^{\Phi}$ (set theoretic difference). The norm of $\nu$ in $B_{\psi}(\mu)$ is the total variation $|\nu|(\Omega)$. [Since $\widetilde{L}^{\Phi}$ is a lattice, it is seen that $B_{\psi}(\mu)$ is a normed linear space, and its completeness is a consequence of the next result.]

With the above definition, $\left(\mathscr{N}^{\oplus}\right)^{*}$ may be characterized as follows.

THEOREM 3. The space $\left(\mathscr{N}^{\oplus}\right)^{*}$ is isometrically equivalent to $B_{\Psi}(\mu)$. More explicitly for each $x^{*} \in\left(\mathscr{N}^{-\oplus}\right)^{*}$ there is a unique $\nu \in B(\mu)$ such that

$$
x^{*}(\hat{f})=\int_{\Omega} f d \nu, \quad \hat{f} \in \mathscr{N}^{\Phi}, f \in \hat{f},
$$

and

$$
\left\|x^{*}\right\|=|\nu|(\Omega) \text {, }
$$

where the integral in (3.12) is defined as that in (3.11).

Proof. With each $x^{*} \in\left(\mathscr{N}^{\Phi}\right)^{*}$, by Lemma 9 and the following remark, there exists a $\nu \in B_{\Psi}(\mu)$ satisfying (3.13). For this proof it may and will be assumed $x^{*} \geqq 0$ so that $\nu \geqq 0$. Then with this $\nu$ let the functional defined by (3.11) be $\widetilde{x}^{*}$, i.e., $\widetilde{x}^{*}(\hat{f})=\int_{\Omega} f d \nu, \widetilde{x}^{*} \geqq 0$. It is claimed that $\widetilde{x}^{*}=x^{*}$. For, by definition of (3.11),

$$
\widetilde{x}^{*}(\hat{f})=\lim _{\pi} \sum_{i=1}^{n} d\left(\hat{f}_{E}\right) \mathcal{\nu}\left(E_{i}\right)=\lim _{\pi} \sum_{i=1}^{n} d\left(\hat{f}_{E_{i}}\right)\left\|x_{E_{i}}^{*}\right\| \geqq x^{*}(\hat{f})
$$

for all $\hat{f} \in \mathscr{N}^{\oplus}$. Hence $\widetilde{x}^{*} \geqq x^{*}$. But the above inequality and Lemma 10 imply

$$
x^{*}(\hat{f}) \leqq \widetilde{x}(\hat{f}) \leqq d(\hat{f}) \nu(\Omega) \leqq d(\hat{f})\left\|x^{*}\right\|, \quad \hat{f} \in \mathscr{N}^{\oplus} .
$$

So $\left\|x^{*}\right\|=\left\|\tilde{x}^{*}\right\|$ and an application of (2.13) to $\tilde{x}^{*}-x^{*} \geqq 0$ yields $\left\|\tilde{x}^{*}-x^{*}\right\|=\left\|\tilde{x}^{*}\right\|-\left\|x^{*}\right\|=0$. Thus $\tilde{x}^{*}=x^{*}$ and the proof is complete in one direction.

Conversely, let $\nu_{0} \in B_{v}(\mu)$ and, for convenience $\nu_{0} \geqq 0$. Then (3.12) defines $0 \leqq x_{0}^{*} \in\left(\mathscr{N}^{-\phi}\right)^{*}$. If $\nu_{0}$ is nontrivial, then $x_{0}^{*} \neq 0$ and $\left\|x_{0}^{*}\right\| \leqq$ $\nu_{0}(\Omega)$. It is to be shown that there is equality here. Let $\pi=$ $\left(E_{1}, \cdots, E_{n}\right)$ be a partition of $\Omega$ such that $\nu_{0}\left(E_{i}\right)>0$, all $i$. Then there exist $f_{i} \in \widetilde{L}^{\Phi}-\mathscr{C}^{\Phi}$ with supports $E_{i}, i=1, \cdots, n$. (This is an immediate consequence of the definition of $B_{q}(\mu)$.) Let $f=f_{1}+$ $\cdots+f_{n}=\mathbf{V}_{i=1}^{n} f_{i}$. So $f \in \widetilde{L}^{\Phi}-\mathscr{C}^{\Phi}$ and $d\left(\hat{f}_{E_{i}}\right)=1$. Also $d(\hat{f})=$ $\max \left\{d\left(\hat{f}_{i}\right), i=1, \cdots, n\right\}=1$, since $\mathscr{N}^{\Phi} \subset\left(\mathscr{N}^{-\Phi}\right)^{* *}$ is an abstract $M$ - 
space. This is also immediate from the result that $\mathscr{N}^{\infty}$ was seen to be isometric to a closed subspace of the abstract $M$-space $L^{\infty}$.l Thus $\sum_{i=1}^{n} d\left(\hat{f}_{E_{i}}\right) \nu_{0}\left(E_{i}\right)=\nu_{0}(\Omega)$ and refining the partition $\pi$ on the left yields $x_{0}^{*}(\hat{f})=\nu_{0}(\Omega)$. It follows that $\left\|x_{0}^{*}\right\|=\nu_{0}(\Omega)$. This proves (3.13).

Uniqueness is simple. If $\nu_{1}$ and $\nu_{2}$ in $B_{\psi}(\mu)$ represent the same $x^{*}$ in $\left(, \gamma^{*}\right)^{*}$, then the above argument, leading to (3.13), shows $\|0\|=\left|\nu_{1}-\nu_{2}\right|(\Omega)$ so that $\nu_{1}=\nu_{2}$. Thus the proof of the theorem is complete.

Proof of Equation (3.8). If $0<z^{*} \in\left(V^{* \Phi}\right)^{*}$ and $\nu$ is the corresponding (positive) element of $B_{\psi}(\mu)$, then by the first part of the proof of Theorem $3, z^{*}(\hat{g})=\int_{\Omega} g d \nu, \hat{g} \in \mathscr{N}^{\oplus}$. But by the second part there exists (not necessarily uniquely) $\hat{0} \leqq \hat{f} \in N^{\Phi}$ such that $d(\hat{f})=1$ and $z^{*}(\hat{f})=\nu(\Omega)=\left\|z^{*}\right\|$. Moreover, if $E \in \Sigma$, then $z_{E}^{*}(\hat{f})=z^{*}\left(\hat{f}_{E}\right)$. If $\nu_{E}$ is the restriction of $\nu$ to $E$, and $\widetilde{z}^{*}$ is the corresponding element of $\left(. V^{\oplus}\right)^{*}$ defined by $\widetilde{z}^{*}(\hat{g})=\int_{\Omega} g d \nu_{E}$, then $\left\|\tilde{z}^{*}\right\|=\nu_{E}(\Omega)=\nu(E)$. But one also has (by the same argument),

$$
\widetilde{z}^{*}(\hat{g})=\int_{\Omega} g d \nu_{L}=\int_{\Omega} g_{E} d \nu=z^{*}\left(\widehat{g}_{E}\right)=z_{E}^{*}(\hat{g}), \quad \hat{g} \in \mathscr{N}^{\Phi} .
$$

It follows that $\widetilde{z}^{*}=z_{E}^{*}$, so that

$$
\left\|z_{E}^{*}\right\|=\nu(E)=\int_{\Omega} f_{E} d \nu=z^{*}\left(\widehat{f}_{E}\right)=z_{E}^{*}(\hat{f}) .
$$

This completes the proof of (3.8).

REMARK. (3.13) can also be proved as follows. By the definition of the integral in (3.11), or (3.12), $x^{*}$ represents a linear functional on a closed subspace of $L^{\infty}$ corresponding to $\mathscr{N}^{\Phi}$, by the normpreserving correspondence $\hat{f} \rightarrow \tilde{f}_{\pi}$. But then by known results about $L^{\infty}$, it follows that $\left\|x^{*}\right\|=|\nu|(\Omega)$. (In this case, however, the argument of the proof of the theorem should be given in establishing the equation (3.8).)

Before the general representation of the elements of $\left(L^{\Phi}\right)^{*}$, an auxiliary result will be proved. As a consequence one will note the useful fact that $\left(L^{\Phi}\right)^{*}$ admits of a decomposition into complementary subspaces (cf., [3], p. 120). One of them may be identified with $\left(\mathbb{C l}^{q}\right)^{\perp}$.

Proposition 2. Every element $x^{*} \in\left(L^{\Phi}\right)^{*}$ can be uniquely decomposed as $x^{*}=y^{*}+z^{*}$ where $y^{*}(f)=\int_{\Omega} f d G, f \in L^{\phi}$, for some $G \in A_{\psi}(\mu)$, and is absolutely continuous, and $z^{*} \in\left(\mathscr{C l}^{\Phi}\right)^{\perp}$. 
Proof. Let $\Sigma_{1} \subset \Sigma$ be the ring of sets of finite $\mu$-measure as before. If $E \in \Sigma_{1}$, let $x^{*}\left(\chi_{E}\right)=G(E)$. Then by the second half of the proof of Theorem $1, G \in A_{\Psi}(\mu)$. So as in that proof one can define $y^{*}(f)=\int_{Q} f d G, f \in L^{\Phi}$. Then $y^{*} \in\left(L^{\varphi}\right)^{*}$ and is absolutely continuous. Letting $z^{*}=x^{*}-y^{*}$, one notes that $z^{*} \in\left(\mathbb{C}^{\Phi}\right)^{\perp}$, and $x^{*}=y^{*}+z^{*}$ is a decomposition of the required type.

To prove uniqueness, let $x^{*}=y_{1}^{*}+z_{1}^{*}$ be another such decomposition, so that $y_{1}^{*}(f)=\int_{\Omega} f d G_{1}, f \in L^{\Phi}$, and $G_{1} \in A_{y}(\mu)$. Since $y_{1}^{*}+x_{1}^{*}=$ $y^{*}+z^{*}$, it implies

$$
y^{*}(f)-y_{1}^{*}(f)=\int_{\Omega} f d\left(G-G_{1}\right)=z_{1}^{*}(f)-z^{*}(f)=0, \quad f \in \mathscr{L}^{\Phi} .
$$

Since $G-G_{1} \in A_{\Psi}(\mu)$, it follows from Theorem 1 above (cf. (3.7)) that $\left\|\left|G-G_{1}\left\|_{\psi}=\right\|\left(y^{*}-y_{i}^{*}\right)\right| \mathscr{C}^{\Phi}\right\|=0$ where $y^{*} \mid \mathscr{C}^{\Phi}$ is the restriction of $y^{*}$ to $\mathscr{C}^{\Phi}$. Thus $G=G_{1}$ and hence $y^{*}=y_{1}^{*}$ and $z^{*}=z_{1}^{*}$. Thus the decomposition is unique and the proposition is proved.

REMARK. Professor A. C. Zaanen has remarked that the above result is also a consequence of general theorems on normed vector lattices. Thus if $x^{*} \in\left(L^{\Phi}\right)^{*}$ then $x^{*}=y^{*}+z^{*}$ uniquely on $L^{\Phi}$, where $z^{*} \in\left(\mathscr{L}^{\Phi}\right)^{\perp}$ and $\left|y^{*}\right| \wedge\left|z^{*}\right|=0$, in the notation of Lemma 6. I wish to thank Professor Zaanen for this and other helpful comments.

Definition 6. Let $\mathscr{A} \Phi(\mu)=A_{\Phi}(\mu) \oplus B_{\Phi}(\mu)$, with norm

$$
\|G\|_{\phi}^{\prime}=\left\||| G_{1}\right\|_{\Phi}+\left|\nu_{1}\right|(\Omega), \quad G \in \mathscr{A}_{\Phi}(\mu),
$$

where $G=G_{1}+\nu_{1}, G_{1} \in A_{\varphi}(\mu), \nu_{1} \in B_{\oplus}(\mu)$. Replacing $\Phi$ by $\Psi$, the corresponding definition gives $\mathscr{A}_{w}(\mu)$. $\left[\|\| \cdot \mid \|_{\infty}\right.$ is given by (3.2).]

Combining all the preceding results $\left(L^{\Phi}\right)^{*}$ can be characterized completely, and the result is given in the following.

THEOREM 4. Let $\Phi, \Psi$ be an arbitrary pair of Young's complementary functions and $L^{\Phi}, \mathscr{A}_{\Psi}(\mu)$ be the Orlicz space on $(\Omega, \Sigma, \mu)$ and the space of additive set functions (given in Definition 6). Then $\left(L^{\Phi}\right)^{*}$ and $\mathscr{A}_{*}(\mu)$ are isometrically isomorphic. More explicitly, for each $x^{*} \in\left(L^{\oplus}\right)^{*}$, there exists a unique $G \in \mathscr{A}_{\Psi}(\mu)$ such that

$$
x^{*}(f)=\int_{\Omega} f d G\left(=\int_{\Omega} f d G_{1}+\int_{\Omega} f d \nu_{1}\right), \quad f \in L^{\Phi},
$$

and

$$
\left\|x^{*}\right\|=\|G\|_{\Psi}^{\prime}\left(=\left\|G_{1}\right\|_{\Psi}+\left|\nu_{1}\right|(\Omega)\right) .
$$

This result is an immediate consequence of Theorems 1 and 3, and Proposition 2. If $\mu(\Omega)<\infty$, this can be specialized as follows. 
CoRollary 4.1. If $(\Omega, \Sigma, \mu)$ is a finite measure space then (3.15) and (3.16) can be written uniquely as

$$
x^{*}(f)=\int_{\Omega} f g d \mu+\int_{\Omega} f d \nu_{0}+\int_{\Omega} f d \nu_{1}, \quad f \in L^{\Phi},
$$

and

$$
\left\|x^{*}\right\|=N_{\Psi}(g)+\left|\nu_{0}\right|(\Omega)+\left|\nu_{1}\right|(\Omega),
$$

where $g \in L^{q}, \nu_{0}$ is a purely finitely additive set function and $\nu_{1} \in B_{y}(\mu)$. Moreover, $\nu_{0}=0$ if $\Phi$ is continuous and $\nu_{1}=0$ if $\Phi$ is discontinuous.

Proof. If $\mu(\Omega)<\infty$ then $A_{\Psi}(\mu) \subset b a(\Omega, \Sigma, \mu)$. Hence by the YosidaHewitt Theorem $\left([6]\right.$, p. 163), $G_{1}$ of (3.15) can be uniquely decomposed into a completely additive $G_{1}^{\prime}$ and a purely finitely additive $\nu_{0}$. Then with the Radon-Nikodým Theorem and ([19], Lemma 5), (3.17) and (3.18) above follow from (3.15) and (3.16) at once.

The last statement follows from the fact that for discontinuous $\Phi$, by Lemma 2 above, $\mathscr{l}^{\Phi}=L^{\Phi}$ so $\mathscr{N}^{\Phi}=\{0\}$ (or $\nu_{1}=0$ ), and for continuous $\Phi$, since $G_{1} \in A_{w}(\mu)$, by Lemma $8 G_{1}$ must be countably additive so that $\nu_{0}=0$, as desired.

REMARK. The result of Theorem 4 has the following interpretation. Even though $L^{\oplus}$ need not admit a (nontrivial) direct sum decomposition into $\mathscr{C l}^{\Phi}$ and a complementary subspace, $\left(L^{\oplus}\right)^{*}$ admits such a decomposition into $\left(\mathbb{C}^{\phi}\right)^{\perp}$ and an appropriate subspace. These are isometrically isomorphic to $B_{\psi}(\mu)$ and $A_{\psi}(\mu)$ respectively.

If the measure space and the Young's function are slightly restricted then $\left(\mathscr{C l}^{\phi}\right)^{\perp}$, or equivalently $\left(\mathscr{N}^{\oplus}\right)^{*}$, can be given a different characterization without involving $B_{v}(\mu)$. The following result, when $\mu(\Omega)<\infty$, was proved by Andô [1]with a different argument.

Theorem 5. Let $x^{*} \in\left(L^{\Phi}\right)^{*}, \mathscr{C}^{\Phi} \neq L^{\Phi}$ and either (i) $\mu$ is localizable (or $\sigma$-finite), or (ii) $M^{\psi}=L^{w}$. If $\Phi$ and $\Psi$ are continuous, then $x^{*} \in\left(\mathscr{l l}^{\Phi}\right)^{\perp}$ if

$$
\sup \left\{\left|x^{*}(f)\right|:\|f\|_{\Phi} \leqq 1\right\}=\sup \left\{\left|x^{*}(f)\right|: M_{\phi}(f) \leqq 1\right\} .
$$

On the other hand, if $x^{*} \in\left(\mathscr{C l}^{\oplus}\right)^{\perp}$ then (3.19) always holds without any other conditions.

Proof. By Theorem 3 (and Lemmas 3, 9, and 10) if $x^{*} \in\left(\mathbb{C l}^{\Phi}\right)^{\perp}$ then both sides of (3.19) are equal to $|\nu|(\Omega)$ so that (3.19) holds whatever $\mu$ is and $\Phi$ is since no special use of the norms (2.3) and 
(2.4) was needed in determining (3.8) and (3.9) which are invoked here. However, all the hypothesis will be used for the converse.

Let (3.19) hold. If $x^{*}=x_{1}^{*}+x_{2}^{*}$ is the decomposition of Proposition 2, then using the fact that $x_{2}^{*} \in\left(\mathscr{A}^{\Phi}\right)^{\perp}$ it follows that (3.19) implies

$$
\sup \left\{\left|x_{1}^{*}(f)\right|:\|f\|_{\Phi} \leqq 1\right\}=\sup \left\{\left|x_{1}^{*}(f)\right|: N_{\Phi}(f) \leqq 1\right\} .
$$

But by Theorem $1, x_{i}^{*}(f)=\int_{\Omega} f d G_{1}$ uniquely and since $\Phi$ is continuous, by Lemmas 2 and $8, M^{\Phi}=\mathscr{M}^{\Phi}$ and $G_{1}$ is countably additive, so when the hypothesis ((i) and (ii)) holds one has $x_{1}^{*}(f)=\int_{\Omega} f g d \mu$ for a unique $g \in L^{\Psi}$, (cf. [16], p. 336, and also [19], Th. 4). Moreover, as in the proof of (3.5), $\sup \left\{\left|x_{1}^{*}(f)\right|:\|f\|_{\Phi} \leqq 1\right\}=N_{\Psi}(g)$. This together with (3.20) and (2.3) yields $\|g\|_{\Psi}=N_{\Psi}(g)$. But $\Phi$ and $\Psi$ are continuous, so by Lemma 1 of this paper it follows that $g=0$, a.e. Hence $x^{*}=x_{2}^{*}$, and the proof is complete.

4. Orlicz spaces with generalized Young's functions. In this section the characterization of $\left(L^{\Phi}\right)^{*}$, with $\Phi$ as a generalized Young's function, extending some results of the preceding section will be considered. The reasons for separating these results from those of $\S 3$ are: in most applications it is the Young's functions (instead of the generalized ones) that appear and moreover the subtleties of the theory are presented there (the whole work of [13] considers only continuous Young's pairs), and the combination would have complicated many of the statements. Note that most of the lemmas of $\S 2$ hold for generalized Young's functions also, and they will be used below.

The result on absolutely continuous functionals can be formulated as

THEOREM 6. Let $\Phi$ be a generalized Young's function and $\mathscr{L}^{\Phi}$ be the closed subspace of $L^{\Phi}$, spanned by the step functions. Then for each $x^{*} \in\left(\mathscr{C}^{\Phi}\right)^{*}$, the adjoint space of $M^{\Phi}$, there exists a unique $G \in A_{\mathbb{w}}(\mu)$, where $A_{\mathbb{T}}(\mu)$ is the space given in Definition 4 , such that

$$
x^{*}(f)=\int_{\Omega} f d G, \quad f \in \mathscr{C}^{\Phi}
$$

and

$$
\left\|x^{*}\right\|=\|\| G \|_{w}
$$

Proof. If $\Phi$ is a Young's function this is Theorem 1. The only new case here is if $\Phi$ is continuous but vanishes on an interval $(0, a)$, $a>0$ so that $L^{\infty} \subset \mathscr{M}^{\Phi} \subset L^{\Phi}$. In this case $\mathscr{M}^{\Phi}$ is clearly the $L^{\Phi}$ - 
closure of $L^{\infty}$. Then, if $G \in A_{\psi}(\mu)$ so that $G$ is an additive set function, by the definition of $\|G\|_{\Psi},(4.1)$ and (4.2) obtain at once.

On the other hand if $G$ is defined, for $x^{*} \in\left(\mathbb{C}^{\oplus}\right)^{*}$, by

$$
G(E)=x^{*}\left(\chi_{E}\right), \quad E \in \Sigma,
$$

then $G$ is additive, vanishes on $\mu$-null sets and, using (2.5),

$$
\begin{aligned}
|G(E)| & \leqq 2\left\|x^{*}\right\| \cdot N_{\phi}\left(\chi_{E}\right) \\
& \leqq 2\left\|x^{*}\right\| \cdot\left[\Phi^{-1}\left(\frac{1}{\mu(E)}\right)\right]^{-1} \leqq 2\left(\left\|x^{*}\right\| / a\right)<\infty .
\end{aligned}
$$

Hence

$$
\sup \{|G(E)|: E \in \Sigma\} \leqq \frac{2}{a}\left\|x^{*}\right\|,
$$

so that by ([6], p. 97), $|G|(\Omega)=v(G)<\infty$. Then by ([6], p. 296),

$$
x^{*}(f)=\int_{\Omega} f d G, \quad f \in L^{\infty}
$$

holds. Since $L^{\infty}$ is dense in $\mathscr{C}^{\Phi},(4.1)$ follows. Moreover

$$
\||G|\|_{\Psi}=\sup \left\{\left|x^{*}(f)\right|:\|f\|_{\Phi} \leqq 1, f \in \mathscr{C}^{\Phi}\right\}=\left\|x^{*}\right\|,
$$

which is (4.2). As the uniqueness is clear, the proof is complete.

REMARK. If $\Phi$ vanishes on an interval $(0, a), a>0$, then the above proof shows that $A_{w}(\mu)$ contains the set of all bounded additive set functions on $\Sigma$ vanishing on $\mu$-null sets whether $\Phi$ jumps or not. If also $\Phi$ jumps $L^{\infty}=\mathscr{L}^{\Phi}=L^{\Phi}$ also holds and this is not generally true otherwise.

Corollary 6.1. If $\Phi, \Psi$, and $A_{\Psi}(\mu)$ are as in the theorem, then the integral (4.2) can be extended to all of $L^{\Phi}$, i.e., for each $G \in A_{\Psi}(\mu)$ one has

$$
x^{*}(f)=\int_{\Omega} f d G, \quad f \in L^{\Phi},
$$

and

$$
\left\|x^{*}\right\|=\|G\|_{\psi}
$$

Proof. By the theorem the integral is defined on $\mathscr{M}^{\Phi}$ for $G \in A_{\Psi}(\mu)$. So let $f \in L^{\Phi}$ be arbitrary. It is no restriction to assume $f \geqq 0$. (The only new case is when $\Phi$ is continuous.) Then there exists a sequence of step functions $0 \leqq f_{n} \uparrow f$ (cf. [9], p. 85), and 
$f_{n} \in \mathscr{H}^{\oplus}$. Hence

$$
\left|x_{1}^{*}\left(f_{n}\right)\right|=\left|\int_{\Omega} f_{n} d G\right| \leqq\left\|f_{n}\right\|_{\odot}\||G|\|_{\psi} \leqq\|f\|_{\varnothing}\|G\|_{\psi}=\alpha \text { (say) }
$$

by (4.1) and (3.2). Since by definition it also follows easily that the norms of the set functions $G$ and its variation $|G|\left(=G^{+}+G^{-}\right.$, the positive and negative parts) are equal, the $G$ may also be assumed in (4.6) to be positive. Then $x_{1}^{*}\left(f_{n}\right) \leqq x_{1}^{*}\left(f_{n+1}\right) \leqq \alpha$. If

$$
\lambda_{n}(E)=\int_{E} f_{n} d G, \quad E \in \Sigma,
$$

then $\lim _{n \rightarrow \infty} \lambda_{n}(E)$ exists for each $E$, and if $\lambda_{0}(E)$ is the limit then $\lambda_{0}$ is additive on $\Sigma$ and $\lambda_{n}$ is clearly $G$-continuous (as well as $\mu$-continuous since $\Phi$ is continuous). So by ([2], p. 343 or p. 345) one may write (4.7) as

$$
\lambda_{0}(E)=\lim _{n \rightarrow \infty} \int_{E} f_{n} d G=\int_{E} f d G \leqq \alpha<\infty, \quad E \in \Sigma .
$$

By linearity then one has

$$
\left|x^{*}(f)\right|=\left|\int_{\Omega} f d G\right| \leqq\|f\|_{\Phi}\|G\|_{\Psi}, \quad f \in L^{\Phi},
$$

so (4.4) holds and $\left\|x^{*}\right\| \leqq\|G\|_{\psi}$. Since the opposite inequality is obvious, (4.5) holds as desired.

If $\Phi$ is a generalized Young's function as above and $\mu(\Omega)=\infty$, then even if $\Phi$ is continuous, there exists $E_{n} \in \Sigma, E_{n} \supset E_{n+1}, \bigcap E_{n}=$ $\varnothing, \mu\left(E_{n}\right)=\infty$, so that $\chi_{E_{n}} \in \mathscr{C}^{\Phi}, N_{\phi}\left(\chi_{E_{n}}\right)=1 / a, a>0$, all $n$. So the norm in $\mathscr{C}^{\Phi}$ is not absolutely continuous, and $L^{\Phi}$ cannot be reflexive in this case whatever other growth conditions are satisfied for $\Phi$ (cf. [8], p. 205). This and Proposition 1 imply the following result.

COROLlaRY 6.2. If $\Phi$ is a generalized Young's function that is not an ordinary (in the sense of Definition 1) Young's function then $L^{\Phi}$ need not be reflexive. It is reflexive if and only if $L^{\Phi}=M^{\Phi}$ and $L^{\Psi}=M^{w}$ where $\Psi$ is complementary to $\Phi$.

The relation between the ordinary and generalized Young's functions is further illustrated by the following

LeMma 11. If $\Phi_{0}$ is a continuous generalized Young's function then there exists a continuous (ordinary) Young's function $\Phi$, such that $\Phi_{0}$ is the principal part of $\Phi$, i.e., $\Phi(x)=\Phi_{0}(x)$ for $x \geqq x_{0}$, for some $x_{0}>0$. 
Proof. The proof is a modification of that given in ([13], p. 16) in which $\Psi$, the complementary function of $\Phi$, was also assumed continuous. Let $x_{1}>0$ be chosen such that $\Phi_{0}(x)>0$ for $x \geqq x_{1}$. Since every continuous convex function is absolutely continuous, $\Phi_{0}^{\prime}(x)$ exists, $>0$, for almost all $x>x_{1}$ and

$$
\Phi_{0}(x)=\Phi_{0}\left(x_{1}\right)+\int_{x_{1}}^{x} \Phi_{0}^{\prime}(t) d t .
$$

Let $x_{2}>x_{1}+1$ be chosen such that $x_{2} \Phi_{0}^{\prime}\left(x_{2}\right)>\Phi_{0}\left(x_{1}\right)+x_{1} \Phi_{0}^{\prime}\left(x_{1}\right)$. This is clearly possible since $\Phi_{0}(x) \rightarrow \infty$ as $x \rightarrow \infty$ and $\Phi_{0}^{\prime}\left(x_{2}\right) \geqq \Phi_{0}^{\prime}\left(x_{1}\right)>0$. It follows, from (4.9), that

$$
\begin{aligned}
\Phi_{0}\left(x_{2}\right)-\Phi_{0}\left(x_{1}\right) & =\int_{x_{1}}^{x_{1}+1} \Phi_{0}^{\prime}(t) d t+\int_{x_{1}+1}^{x_{2}} \Phi_{0}^{\prime}(t) d t \\
& \leqq \Phi_{0}^{\prime}\left(x_{1}+1\right)+\Phi_{0}^{\prime}\left(x_{2}\right)\left(x_{2}-x_{1}-1\right) .
\end{aligned}
$$

So $\Phi_{0}\left(x_{2}\right)<x_{2} \Phi_{0}^{\prime}\left(x_{2}\right)$. Setting $\alpha=x_{2} \Phi_{0}^{\prime}\left(x_{2}\right) / \Phi_{0}\left(x_{2}\right)$, one notes that $\alpha>1$ and if

$$
\Phi(x)= \begin{cases}\Phi_{0}\left(x_{2}\right) \cdot\left(\frac{|x|}{x_{2}}\right)^{\alpha}, & \text { for }|x| \leqq x_{2} \\ \Phi_{0}(x), & \text { for }|x| \geqq x_{2},\end{cases}
$$

then $\Phi(\cdot)$ is a continuous Young's function with $\Phi_{0}$ as its principal part for $x \geqq x_{0}=x_{2}>0$, completing the proof.

REMARK. It follows from this lemma and ([13], p. 110), that there is no need to distinguish between the Definitions 1 and 2 if $\mu(\Omega)<\infty$. However, if $\mu(\Omega)=\infty$, the distinction should be maintained. If $\mathscr{N}^{\Phi}=L^{\Phi} / \mathscr{L}^{\Phi}$ where $\mathscr{C}^{\Phi}$ is the closed subspace spanned by the step functions of $L^{\Phi}$, and if every simple function is replaced by a step function in the work of $\S 3$ from Lemma 9 onwards, (since no special properties of $\Phi$ are used) then it follows that Theorem 3 holds for $\mathscr{N}^{\oplus}$ as defined here. In view of Corollary 6.1 above, Proposition 2 also holds. Consequently Theorem 4 takes the following form:

THEOREM 7. Let $\Phi, \Psi$ be an arbitrary complementary pair of generalized Young's functions and $L^{\Phi}, \mathscr{A}_{\psi}(\mu)$ be the Orlicz space on $(\Omega, \Sigma, \mu)$ and the space of additive set functions (given in Definition 6 , with $\Phi, \Psi$ as the generalized functions as here) then for each $x^{*} \in\left(L^{\Phi}\right)^{*}$, there exists a unique $G \in \mathscr{A}_{\Psi}(\mu)$ such that

$$
x^{*}(f)=\int_{\Omega} f d G\left(=\int_{\Omega} f d G_{1}+\int_{\Omega} f d \nu_{1}\right), \quad f \in L^{\Phi}
$$

where $G_{1} \in A_{\Psi}(\mu), \nu_{1} \in B_{\Psi}(\mu), G=G_{1}+\nu_{1}$, and 


$$
\left\|x^{*}\right\|=\|G\|_{\psi}^{\prime}\left(=\left|\left\|G_{1}\right\|_{\psi}+\right| \nu_{1} \mid(\Omega)\right) \text {. }
$$

This result is a consequence of Theorems 3 and 6 .

An alternate characterization of singular functionals, given in Theorem 5 for Young's functions need not hold if $\mu(\Omega)=\infty$. The intervening results, Lemmas 1,8 and others used there, are not available now. Some subsidiary results will be presented in the next section.

5. Miscellaneous results. It is well-known that a Hahn-Banach extension of a continuous linear functional from a proper subspace to the whole Banach space need not be unique. In these circumstances the following result is of interest.

Proposition 3. Let $\Phi$ be a (generalized) Young's function. Then every $x^{*} \in\left(\mathscr{C l}^{\oplus}\right)^{*}$ has a unique Hahn-Banach (i.e., norm preserving) extension to all of $L^{\Phi}$.

Proof. If $x^{*} \in\left(\mathscr{C l}^{\Phi}\right)^{*}$, then by Theorem 6 there is a unique $G \in A_{\mathbb{F}}(\mu)$ such that for all $f$ in $\mathscr{C l}^{\Phi}$,

$$
x^{*}(f)=\int_{\Omega} f d G, \quad\left\|x^{*}\right\|=\|G\|_{\psi} .
$$

But the integral in (5.1) is defined for all $f \in L^{\oplus}$ and the norm equation holds (cf. Corollary 6.1). Consequently if $x_{1}^{*}$ is the thus obtained extension on $L^{\Phi}$, it is a Hahn-Banach extension. If $x_{2}^{*}$ is any other such extension of $x^{*}$, let $z_{1}^{*}=x_{2}^{*}-x_{1}^{*}$ so that $z_{1}^{*} \in\left(\mathscr{C}^{\Phi}\right)^{\perp}$, and $x_{2}^{*}=$ $x_{1}^{*}+z_{1}^{*}$ is a decomposition of the type described in Proposition 2. Also by the first part $\left\|x_{2}^{*}\right\|=\left\|x_{1}^{*}\right\|=\left\|x^{*}\right\|$. But by Theorem 7 , it follows that

$$
\left\|x_{2}^{*}\right\|=\left\|x_{1}^{*}\right\|+|\nu|(\Omega)
$$

for a unique $\nu$ in $B_{q}(\mu)$ corresponding to $z_{1}^{*}$. However, the equations in the preceding two lines imply $|\nu|(\Omega)=0$ so that $z_{1}^{*}=0$ by Theorem 3 . Consequently $x_{1}^{*}=x_{2}^{*}$, as was to be shown.

In $\S 3$ the concept of continuity in the extended sense was considered in discussing purely discontinuous Young's functions. In that connection the following result can be proved.

Proposition 4. Let $\Phi, \Psi$ be a (generalized) Young's complementary pair such that $\Psi$ is continuous in the extended sense (i.e., $\Psi$ and $\Psi^{\prime}$ are continuous on $0 \leqq x \leqq x_{0}$, for some $x_{0}>0, \Psi^{\prime}(x)>0, x>0$, and $\Psi(x)=\Psi^{\prime}(x)=\infty$ for $x>x_{0}$ ). Then $L^{\Phi}$ is rotund (=strictly convex). 
Proof. If $\Psi$ is continuous in the extended sense, then by construction of its complementary function $\Phi$, (cf. [13], [15], [17], or [26]) it follows that $\Phi$ and $\Phi^{\prime}$ are continuous, $\lim _{x \rightarrow \infty} \Phi^{\prime}(x)=x_{0}$, and that $\Phi^{\prime}$ is strictly increasing, so that $\Phi$ is also a strictly convex, continuous Young's function. Under these circumstances, it was proved in ([20], p. 682) that $L^{\Phi}$ is rotund, with the norm (2.4). However, using ([13], Th. 10.4) one can immediately deduce the same conclusion from the foregoing result with norm (2.3) as well. This completes the proof.

REMARK. 1. If $\mu$ is a nonatomic $\sigma$-finite measure then the above result was first established, with norm (2.3), in ([17], Th. 5) where the condition was shown to be necessary. [The nonatomicity is essential for the latter conclusion.]

2. Lemma 1 holds if the complementary function $\Psi$ of $\Phi$ is continuous in the extended sense. This follows from the fact that in this case $\Phi$ is strictly convex and $\Phi^{\prime}$ is continuous and with this, one can show that $\int_{\Omega} \Phi(f) d \mu=1$ if $N_{\mathscr{Q}}(f)=1$, (see [20], p. 682 for a proof). This is the crucial step (2.8) in the proof of that lemma. Note, however, that the conditions of Lemma 1 do not assume that $\Phi^{\prime}$ is continuous. Thus the above comment merely complements, but not generalizes, the result of Lemma 1 in some cases.

Some notes. In the rest of this section some related problems and results will be briefly discussed. With the work of $\S 3$ and $\S 4$, and of [11], a general result on $\left(L^{\phi}\right)^{* *}$, the second conjugate of $L^{\phi}$, can be stated as follows.

Proposition 5. If $\Phi, \Psi$ are complementary functions, $\Phi$ is a continuous Young's function and the measure space $(\Omega, \Sigma, \mu)$ is localizable (cf. [16]) or $\sigma$-finite, then $\left(L^{\Phi}\right)^{* *}$ is isometrically isomorphic to $\mathscr{A}_{\Phi}(\mu)=A_{\phi}(\mu) \oplus B_{\Phi}(\mu) \oplus C_{\Phi}(\mu)$ where $C_{\phi}(\mu)$ is an abstract $(M)$-space, conjugate of $B_{\psi}(\mu)$, and where the norm in the space $\mathscr{A}_{\phi}(\mu)$ is given by: if $H=F+\nu+\delta \in \mathscr{C}_{\Phi}(\mu),\left(F \in A_{\Phi}(\mu), \nu \in B_{\phi}(\mu), \delta \in C_{\phi}(\mu)\right)$ then

$$
\|H\|_{\Phi}^{\prime}=\max \left(\left\|\left|F\left\|_{\phi}+|\nu|(\Omega),\right\| \delta \|\right),\right.\right.
$$

so that $x^{* *} \in\left(L^{\phi}\right)^{* *}$ implies $\left\|x^{* *}\right\|=\|H\|_{\phi}^{\prime}$ for a unique $H \in \mathscr{A}_{\phi}(\mu)$.

Proof. By Theorem 4, $\left(L^{\oplus}\right)^{*}=A_{\psi}(\mu) \oplus B_{\psi}(\mu)$, isometrically. If $\Phi$ is continuous and $\mu$ is localizable or $\sigma$-finite then by ([19], Lemma 5) and by Lemma $8, A_{\psi}(\mu)$ is equivalent to $L^{\Psi}$. Hence, writing equality for isometric equivalence,

$$
\left(L^{\Phi}\right)^{* *}=\left(L^{\Psi}\right)^{*} \oplus\left(B_{\Psi}(\mu)\right)^{*}=A_{\Phi}(\mu) \oplus B_{\Phi}(\mu) \oplus C_{\Phi}(\mu)
$$


by Theorem 6 (since $\Psi$ can be a generalized Young's function this time), and where $C_{\phi}(\mu)$ is the abstract $(M)$-space that is conjugate to the abstract $(L)$-space $B_{\Psi}(\mu)$, given by $([11]$, Th. 15$) . \quad\left[C_{\oplus}(\mu)\right.$ can be given other representations as in [11], but it is omitted here since it is not particularly relevant.] The norm condition is now a standard result. The result follows.

REMARK. If $\Phi$ is not continuous, or $\mu$ is arbitrary then $A_{\Psi}(\mu)$ cannot be identified with $L^{\psi}$. If $\mu(\Omega)<\infty$, and $\Phi$ is discontinuous or $\mu$ is arbitrary and $\Phi$ is purely discontinuous, then $A_{\Psi}(\mu)$ can be identified with $b a(\Omega, \Sigma, \mu)$ and Kakutani's theorem can again be applied to yield abstract $(M)$-spaces to characterize $\left(L^{\Phi}\right)^{* *}$. The few remaining cases are still open.

Instead of using the norms (2.3) and (2.4), only (2.4) can be used for both $L^{\Phi}$ and $L^{\psi}$ with advantage, after the normalization of the Young's functions $\Phi, \Psi$ as $\Phi(1)+\Psi(1)=1$. Its importance was emphasized in [27], and the work in [20] illustrates the resulting economy and convenience in most cases of interest. (2.4) and (2.5) then must be replaced by (5.3) and (5.4) below where $f \in L^{\Phi}$ if and only if $N_{\phi}(f)<\infty$ with

$$
N_{\varphi}(f)=\inf \left\{k>0, \int_{\Omega} \Phi(f) d \mu \leqq \Phi(1)\right\},
$$

and

$$
\Phi(1) N_{\Phi}(f) \leqq\|f\|_{\Phi} \leqq 2 N_{\varphi}(f) .
$$

[If $\Phi(1)=0$, and $\Phi$ is thus discontinuous, by definition, one can directly show that $N_{\Phi}(f)=\|f\|_{\Phi}=\|f\|_{\infty}$ in this case.] Then the result of Theorem 4 holds without change and the theory exhibits some symmetry (cf. [20]) and its analogy with the $L^{p}$-theory is more transparent. (The work through $\S 3$ of this paper was referred to as Part II of [19] in [20], [23] and certain other papers.)

The following problems are related to the work of this paper.

1. The structure of $A_{\phi}(\mu)$ is of interest in view of Proposition 5. If $V^{\Phi}(\mu)$ is the subspace of $A_{\Phi}(\mu)$ of $\mu$-continuous elements and if $\Phi$ is continuous, then Uhl [23] has characterized $\left(V^{\Phi}(\mu)\right)^{*}$ when $\mu$ is only a finitely additive (finite) nonnegative set function.

2. If $\Phi$ satisfies a growth condition (the $A_{2}$-condition of [13]) then considerable work has been done by N. Dinculeanu on $L^{\Phi}$ spaces of vector fields, (cf. [4]). The relaxation of the growth condition is of interest, since, for instance, it has some interesting applications in Probability and Statistics. This may be seen from the recent work of T. S. Pitcher [18] when one recognizes his $E^{p}$ spaces, $1 \leqq p \leqq \infty$, 
to be identifiable with an appropriate space of (totally measurable) vector fields. A more recent and readable account of the latter theory, which is needed here, may be found in [5].

3. The work of $\S 3$ and $\S 4$ also shows that $\mu$ may be assumed to be only finitely additive. If $\mu(\Omega)<\infty$, the corresponding theory of $L^{\Phi}(\mu)$ is obtainable from the recent work of Uhl [23], but it will be of interest to remove this restriction. The corresponding theory for Köthe-Toeplitz and Riesz spaces appears possible with the methods of this paper. A relatively simple representation theorem (when the norm in the given space is absolutely continuous and the measure space is the unit interval, which corresponds to $M^{\Phi}=L^{\Phi}$ above) was obtained in [14], and somewhat more general results were proved in [7]. The general characterization of arbitrary singular linear functionals on these spaces is yet to be completed.

4. (Note added, October 1967.) Using the methods of this paper, Mr. N. E. Gretsky has recently obtained characterizations of the conjugate spaces of a general class of Banach function spaces in his $\mathrm{Ph}$. D. thesis entitled Representation theorems on Banach function spaces, at Carnegie Tech., 1967, and only a few more cases need be settled in this direction.

\section{REFERENCES}

1. T. Andô, Linear functionals on Orlicz spaces, Nieuw Arch. Wisk. 8 (1960), 1-16.

2. R. G. Bartle, A general bilinear vector integral, Studia Math. 15 (1956), 337-352.

3. M. M. Day, Normed Linear Spaces, Springer-Verlag, Berlin, 1958.

4. N. Dinculeanu, Espaces d'Orlicz de champs de vecteurs-IV, Studia Math. 19 (1960), $321-331$.

5. Linear operators on spaces of totally measurable functions, Rev. Roum. Math. Pures et Appl. 10 (1965), 1493-1524.

6. N. Dunford and J. T. Schwartz, Linear operators, Part I: General Theory, Interscience, New York, 1958.

7. H. W. Ellis and I. Halperin, Function spaces determined by a levelling length function, Canad. J. Math. 5 (1953), 576-592.

8. I. Halperin, Reflexivity in the $L^{\lambda}$ function spaces, Duke Math. J. 21 (1954), 205208.

9. P. R. Halmos, Measure theory, D. Van Nostrand Co., New York, 1950.

10. S. Kakutani, Concrete representation of abstract $(L)$-spaces and the mean ergodic theorem, Ann. of Math. 42 (1941), 523-537.

11. - Concrete representation of abstract (M)-spaces, Ann. of Math. 42 (1941), 994-1024.

12. G. Köthe and $\mathrm{O}$. Toeplitz, Lineare Räume mit unendlich vielen Koordinaten und Ringe unendlicher Matrizen, J. Reine Angew. Math. 171 (1934), 193-226.

13. M. A. Kransnosel'skii and Ya. B. Rutickii, Convex Functions and Orlicz Spaces, P. Noordhoff Ltd., Groningen, 1961.

14. G. G. Lorentz and D. G. Wertheim, Representation of linear functionals of Köthe spaces, Canad. J. Math. 5 (1953), 568-575.

15. W. A. J. Luxemburg, Banach function Spaces, Delft, 1955. 
16. E. J. McShane, Families of measures and representations of algebras of operators, Trans. Amer. Math. Soc. 102 (1962), 328-345.

17. H. W. Milnes, Convexity of Orlicz spaces, Pacific J. Math. 7 (1957), 1451-1483.

18. T. S. Pitcher, A more general property than domination for sets of probability measures, Pacific J. Math. 15 (1965), 597-611.

19. M. M. Rao, Linear functionals on Orlicz spaces, Nieuw Arch. Wisk. 12 (1964), 77-98.

20. - Smoothness of Orlicz spaces, Indag. Math. 27 (1965), 671-690.

21. P. C. Rosenbloom, Quelques classes de problémes extrémaux, Bull. Soc. Math. France 79 (1951), 1-58, et 80 (1952), 183-215.

22. A. E. Taylor, Introduction to functional analysis, Wiley, New York, 1958.

23. J. J. Uhl, Jr., Orlicz spaces of finitely additive set fvnctions and set martingales, Ph. D. thesis, Carnegie Tech., 1966.

24. W. H. Young, On classes of summable functions and their Fourier series, Proc. Roy. Soc. (A) 87 (1912), 225-229.

25. A. C. Zaanen, An Introduction to the theory of integration, North-Holland Publishing Co., Amsterdam, 1958.

26. Linear analysis, North-Holland Publishing Co., Amsterdam, 1953.

27. A. Zygmund, Trigonometric series, Vol I (2nd ed.), Cambridge University Press, 1959.

Received February 7, 1967. Supported by NSF grants GP-1349 and GP-5921.

CARNegie Institute of TechNology

Pittsburgh, Pennsylvania 



\title{
PACIFIC JOURNAL OF MATHEMATICS
}

\author{
EDITORS
}

\section{H. ROYDEN}

Stanford University

Stanford, California

\author{
J. P. JANS \\ University of Washington \\ Seattle, Washington 98105
}

\section{J. DugundJI}

Department of Mathematics

University of Southern California

Los Angeles, California 90007

\section{RICHARD ARENS}

University of California

Los Angeles, California 90024

\section{ASSOCIATE EDITORS}
E. F. BECKENBACH
B. H. NEUMANN
F. WOLF
K. YOSIDA

\section{SUPPORTING INSTITUTIONS}

\author{
UNIVERSITY OF BRITISH COLUMBIA \\ CALIFORNIA INSTITUTE OF TECHNOLOGY \\ UNIVERSITY OF CALIFORNIA \\ MONTANA STATE UNIVERSITY \\ UNIVERSITY OF NEVADA \\ NEW MEXICO STATE UNIVERSITY \\ OREGON STATE UNIVERSITY \\ UNIVERSITY OF OREGON \\ OSAKA UNIVERSITY \\ UNIVERSITY OF SOUTHERN CALIFORNIA
}

\author{
STANFORD UNIVERSITY \\ UNIVERSITY OF TOKYO \\ UNIVERSITY OF UTAH \\ WASHINGTON STATE UNIVERSITY \\ UNIVERSITY OF WASHINGTON \\ AMERICAN MATHEMATICAL SOCIETY \\ CHEVRON RESEARCH CORPORATION \\ TRW SYSTEMS \\ NAVAL WEAPONS CENTER
}

Mathematical papers intended for publication in the Pacific Journal of Mathematics should be in typed form or offset-reproduced, double spaced with large margins. Underline Greek letters in red, German in green, and script in blue. The first paragraph or two must be capable of being used separately as a synopsis of the entire paper. It should not contain references to the bibliography. Manuscripts, in duplicate if possible, may be sent to any one of the four editors. All other communications to the editors should be addressed to the managing editor, Richard Arens, University of California, Los Angeles, California 90024.

Each author of each article receives 50 reprints free of charge; additional copies may be obtained at cost in multiples of 50 .

The Pacific Journal of Mathematics is published monthly. Effective with Volume 16 the price per volume (3 numbers) is $\$ 8.00$; single issues, $\$ 3.00$. Special price for current issues to individual faculty members of supporting institutions and to individual members of the American Mathematical Society: $\$ 4.00$ per volume; single issues $\$ 1.50$. Back numbers are available.

Subscriptions, orders for back numbers, and changes of address should be sent to Pacific Journal of Mathematics, 103 Highland Boulevard, Berkeley 8, California.

Printed at Kokusai Bunken Insatsusha (International Academic Printing Co., Ltd.), 7-17, Fujimi 2-chome, Chiyoda-ku, Tokyo, Japan.

PUBLISHED BY PACIFIC JOURNAL OF MATHEMATICS, A NON-PROFIT CORPORATION

The Supporting Institutions listed above contribute to the cost of publication of this Journal, but they are not owners of publishers and have no responsibility for its content or policies. 


\section{Pacific Journal of Mathematics}

\section{Vol. 25, No. $3 \quad$ November, 1968}

Philip Marshall Anselone and Theodore Windle Palmer, Collectively

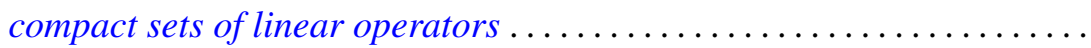

Philip Marshall Anselone and Theodore Windle Palmer, Spectral analysis of collectively compact, strongly convergent operator sequences.........

Edward A. Bender, Characteristic polynomials of symmetric matrices...... 433

Robert Morgan Brooks, The structure space of a commutative locally convex

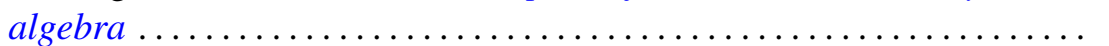

Jacob Feldman and Frederick Paul Greenleaf, Existence of Borel

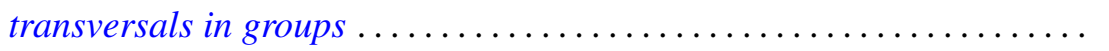

Thomas Muirhead Flett, Mean values of power series 463

Richard Vernon Fuller, Relations among continuous and various

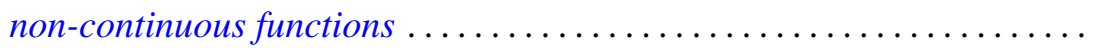

Philip Hartman, Convex sets and the bounded slope condition ............

Marcel Herzog, On finite groups containing a CCT-subgroup with a cyclic Sylow subgroup .

James Secord Howland, On the essential spectrum of Schroedinger

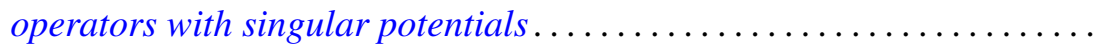

Thomas William Hungerford, On the structure of principal ideal rings .....

Paul Joseph Kelly and Ernst Gabor Straus, Curvature in Hilbert geometries. II mpati Madhusudana Rao, Linear functionals on Orlicz spaces:

Malempati Madhusudana Rao, Linear functionals on Orli
$\quad$ General theory............................
Stanley F. Robinson, Theorems on Brewer sums.......
Ralph Tyrrell Rockafellar, A general correspondence betw

Malempati Madhusudana Rao, Linear functiona
$\quad$ General theory.........................
Stanley F. Robinson, Theorems on Brewer sums
Ralph Tyrrell Rockafellar, A general corresponden

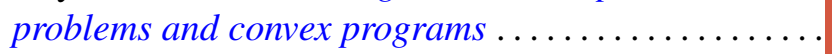

Richard Benjamin Sher, Defining subsets of $E^{3}$ by cubes.

Howard Jacob Weiner, Invariant measures and Cesàro summability... 